\title{
An Investigation on the Dynamics of High-Speed Systems Using Nonlinear Analytical Floating Ring Bearing Models
}

\author{
Athanasios Chasalevris \\ General Electric, Rugby CV212NH, UK \\ Correspondence should be addressed to Athanasios Chasalevris; athanasios.chasalevris@ge.com
}

Received 9 May 2016; Revised 1 July 2016; Accepted 5 July 2016

Academic Editor: Ryoichi Samuel Amano

Copyright ( 2016 Athanasios Chasalevris. This is an open access article distributed under the Creative Commons Attribution License, which permits unrestricted use, distribution, and reproduction in any medium, provided the original work is properly cited.

\begin{abstract}
The scope of this paper is to investigate the dynamics of a rotor-bearing system of high-speed under recently developed analytical bearing models. The development of a theory that can yield the dynamic response of a high-speed system without short/long bearing approximation and without time-consuming numerical methods for the finite-length bearing model is the outcome of this work. The rotor system is introduced as a rigid body so that the dynamics of the system are influenced only from the nonlinear bearing forces which are introduced with closed form expressions. The outcome is a system of nonlinear equations and its solution produces the dynamic response of the high-speed system using exact analytical solution for the bearing forces. The transient dynamic response of the system is evaluated through the wide range of rotating speed and under different bearing solutions including short bearing approximation, presenting the subsynchronous components that are developed when instabilities occur. Time-frequency analysis of the resulting response time-series is presented and the outcome is compared with that obtained from numerical solution of the bearing lubrication and with the short bearing approximation model.
\end{abstract}

\section{Introduction}

Numerous simulations considering different geometric and physical parameters of the floating ring bearing elements can be required in the design of high-speed systems. Unfortunately, the lack of the possibility of expressing the dynamic response of a rotating system in direct relevance to the bearing design parameters leads to numerous case studies considering transient response analysis under various combinations of the bearing geometry, interpreted to variation of bearing radial clearance, and of bearing width through a specified range of values. The nonlinear response of such systems usually contains additional sub/superharmonics that are responsible for leading the system beyond the demands for maximum response amplitude, bearing eccentricities, power losses, acoustic emission, and so forth. More specifically, the developed eccentricities of the bearings are of crucial importance, as they are assumed to be the first indication for system durability considering its millions of run-ups and rundowns in applications of automotive turbochargers.
The variation of bearing design parameters consists of a set of run-up simulations that can consider some hundreds of individual geometric configurations for an initially developed turbosystem with dynamic response and other operational characteristics to be evaluated in each of them. Then, the outcome of that case study, that is, mainly the response time-series at selected points of the rotor-bearing system, is postprocessed with decompositions in time and frequency domain, orbits are plotted at selected speeds, and other operational parameters are extracted for further analysis, to note their tendency regarding each bearing configuration. The high evaluation capacity of modern computers and the implementation of developed numerical tools and theories for rotor-bearing dynamics in commercial software allow simulating such systems and offer confident results in relatively reasonable time.

In a run-up of an automotive turbocharger, three domains of rotational speed usually provoke the three corresponding instabilities at the inner and outer fluid films of the floating ring bearings $[1,2]$. There is also total instability at even 
higher speeds that soon leads to system failure [3]. The three subsynchronous components of the response, referred to as sub-1, sub-2, and sub-3, are developed due to oil whirl/whip instability and are influenced in their amplitude and duration from the bearing's physical and geometric parameters. The channel through which the bearing's geometric and physical configuration influences the bearing's instability is the resulting impedance and friction forces/moments; these phenomena have been extensively explained in [1-4]. Therefore, the model for evaluating oil film pressure distribution and then impedance forces and moments from the fluid film to reaction parts (shaft, ring, and housing) crucially defines the rotational speed in which instability will occur (or not) and the amplitude with which the subsynchronous/subharmonic response will be developed. It is important to remember that the presence and amplitude of the subcomponents are not only influenced by the bearing's properties; various parameters contribute to this, such as unbalance magnitude, elastic component deformations, internal damping mechanisms (material damping, frictional damping between compressor wheel and shaft, etc.), and preloading stresses $[5,6]$.

The possibilities of incorporating floating ring bearing simulation into rotor dynamic algorithms are many and are extensively discussed in the literature. The numerical solution of the Reynolds equation for both inner and outer oil films has been performed with numerical methods such as FDM, FEM, and CFD [7-13]. Such methods are quite accurate but, even after the development of very efficient solvers, they are considered slower in comparison to direct formulas for the bearing's impedance forces yielded from short/long bearing approximation. However, the incorporation of holes in the floating ring connecting the inner and the outer film leaves no room for nonnumerical treatment of the Reynolds full equation. The short/long bearing approximation has been widely used and the literature features many rotor dynamic investigations of turbosystems under this assumption [1420]. However, the length-to-diameter ratio presented in the inner and outer film of the floating ring bearings is not always below or near $L / D=0.5$; in automotive turbochargers, very frequently, the outer fluid film is defined with a ratio near or even higher than $L / D=0.75$.

The short bearing approximation will give a different pressure distribution, especially for high eccentricities and high eccentricity rate of change compared to the pressure distribution evaluated numerically. However, the difference in the final outcome, that is, impedance force, is not really relatively big, so the approximation can still be acceptable for the demands of rotor-bearing design. Considering further the bearing's operating parameters, such as power loss in the lubricant, shearing stresses developed in it, resulting torque from the oil to the ring, and increment of ring speed, the method of the evaluation of pressure distribution can lead to different results regarding the occurrence of instabilities and their characteristics. This paper aims to highlight the evaluated ring speed under different solutions for pressure distribution.

As mentioned above, the evaluation time of bearing forces is an important issue for an efficient design procedure. For a rotor dynamic simulation of, for example, 100 cases of bearing geometric configurations, operating temperatures, and other parameters, the evaluation of transient response can be a matter of days (without postprocessing). Given that a simulation of a transient run-up of an automotive application turbocharger should be performed up to the rotating speed of $250 \mathrm{kRPM}$ or even $300 \mathrm{kRPM}$ and that the resulting response time history should be evaluated for $10 s-15 s$, the length of time histories exceed usually the 1E6 samples (extrapolation is performed before postprocessing) under constant time interval, for example, $1 e-5 \mathrm{~s}$. With a Matlab ${ }^{\circledR}$ Solver ode15s method [21, 22], the bearing routine would be called around 2E7 times (depending on the stiffness of the mathematical system). A subroutine for the bearing's dynamics does not last more than $0.02 \mathrm{~s}$ with an FDM solution (using ADI elimination with relatively weak convergence criteria) and $0.005 \mathrm{~s}$ for a short bearing approximation solution; further details will be given below. The evaluation time dedicated to the bearing routine during a DoE (e.g., 100 cases) can be drastically reduced if the bearing impedance terms are returned faster. Therefore, a suggestion for a floating ring bearing model consisting of analytical closed form expressions would contribute to increasing evaluation speed and considering the finite-length bearing geometry would contribute to the rotor dynamic analysis of high-speed systems in terms of accurate bearing simulation.

This paper does not aim to delve into the mechanisms of instabilities in turbosystems; this issue has been thoroughly discussed in recent $[1-6,11-14]$ and past literature [7-10]. It aims to use two theoretical models for floating ring bearing simulation that are based on a recent achievement of analytical solution of the Reynolds equation in finite-length journal bearings [23-25] and in the application on the floating ring bearings of finite length [26-28]. This analytical solution was implemented on the floating ring bearing with two different theoretical models; the first leads to an exact analytical solution and the second to an approximate analytical solution $[26,27]$. Both theoretical models consist of four SL problems, with one of the four to be treated differently in each of the models. The first theoretical model, the exact analytical solution, uses PSM to solve the one SL problem and to create the resulting pressure distribution in each of the four fluid films (two floating ring bearings are always incorporated). The second, the approximate analytical solution, uses Bessel functions for the solution of the one SL problem. This paper presents shortly the analytical treatment of the Reynolds up to the point that the solution is split in the four SL problems, referring always to the recent work [26]. The solutions for the bearing are incorporated in a rotor dynamic algorithm that simulates a rotor-bearing system consisting of a rigid rotor carrying its components (compressor/turbine wheels) and two floating ring bearings [28]. The motions of the rotor and of the floating rings are expressed using $10 \mathrm{DOF}$ and the set of equations consists of 10 2nd-order ODEs that are converted to 20 1st-order ODEs.

Both theoretical bearing models (exact and approximate) express the impedance forces and moments of the bearing in closed form expressions. The comparison of the system's dynamic behaviour among different bearing models also 


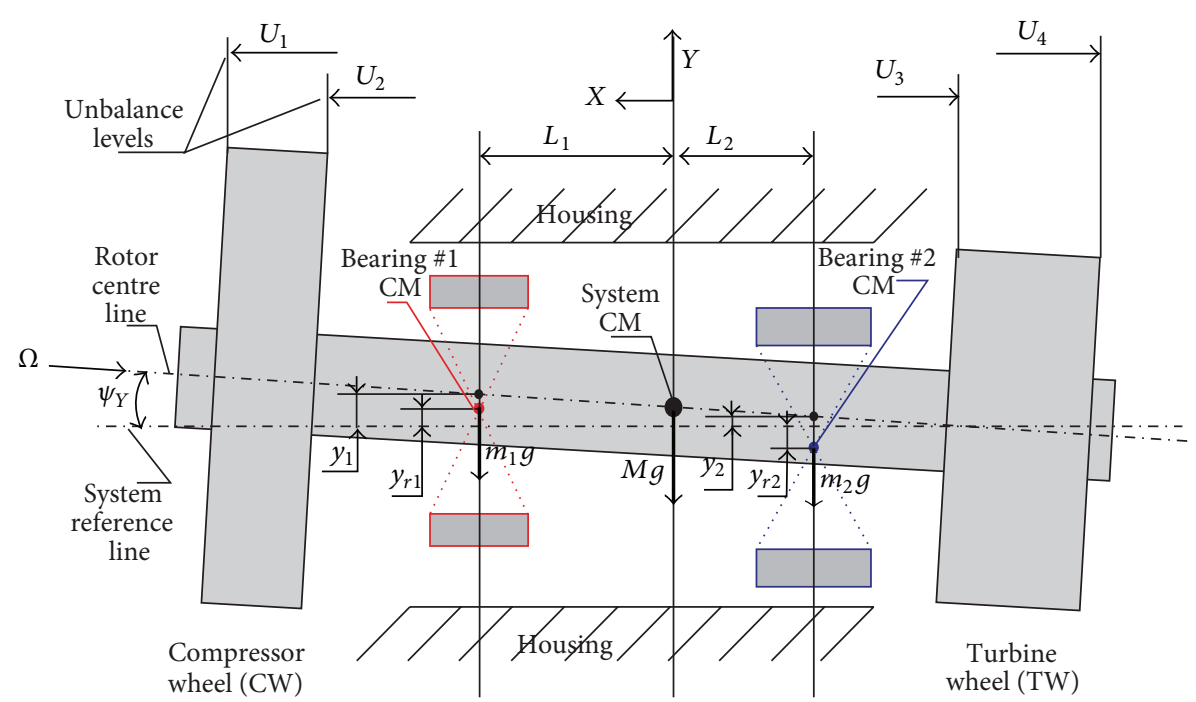

FIGURE 1: Representation and definition of the physical and geometrical parameters of the rotor-bearing system consisting of a rigid rotor carrying two wheels at its ends and mounted on two floating ring bearings.

incorporates results with a short bearing model and results with a finite bearing model solved with FDM.

The results incorporate evaluated time histories for a set of geometric configurations of the rotor-bearing system; two systems are simulated covering the range of dimensions and consequently of operating speeds for the automotive turbochargers of passenger cars. The bearing eccentricities, the compressor wheel response, and the trajectories of the rotor and the floating rings during run-up are of major interest and are compared among different methods of bearing simulation. Time-frequency decomposition of the compressor wheel response is performed in order to extract characteristics of the subsynchronous response. The results among the different bearing models were found either in good agreement to each other or in divergence regarding the instability thresholds and they are discussed extensively. The pressure distribution in the bearing fluid films under various operating conditions that has been compared among the different bearing models [26] is the main source of the differences. The resulting ring speed is also compared as further interpretation of the different instability thresholds of the bearing models.

\section{Motion Equations of the Rotor-Bearing System}

The elastic deformations of a rotor in a high-speed system such as automotive turbochargers do not reach severe magnitudes. This does not mean that the simulation of the rotor as an elastic body is out of any consideration [4, 12, 14]. In this work, in order to highlight the influence of journal bearings on the dynamics of the high-speed system, the rotor is considered as a rigid body [16-18].
In this analysis, a rigid rotor and two rigid wheels, with their masses concentrated at each rotor end, are fixed to each other and rotate at a rotational speed $\Omega$ around the rotor line, as shown in Figure 1. The three components (CW, TW, and rotor) are assumed to have a total mass $M$, a total moment of inertia (polar) $J_{P}$ with respect to the rotor centre line, and a total diametric moment of inertia $J_{T}$ with respect to the vertical and horizontal axes that pass through the centre of mass of the system; see Figure 1. A global rectangular coordinate system $X Y Z$ has its centre at the system reference line at the plane of the centre of mass of the system; see Figure 1. Since the system is not assumed to obtain any axial displacement (hypothesis of plane orbits), the coordinate system $X Y Z$ is fixed and with respect to this system all the lateral displacements will be defined.

Two variables at the vertical plane $X Y$ and two variables at the horizontal plane $X Z$ are used to define the lateral displacement and the tilting angle of the system. The variables $y_{1}$ and $y_{2}$ express the vertical displacement of the system at the locations of the bearings (plane of bearing/ring centre of mass) while the variables $z_{1}$ and $z_{2}$ express the horizontal system displacement. The displacement of the CM is expressed with variables $y_{\mathrm{CM}}$ and $z_{\mathrm{CM}}$ in vertical and horizontal direction correspondingly. Two more variables are introduced to define the tilting angles of the rotating system with respect to the reference line at the vertical and horizontal direction; these are $\psi_{Y}$ and $\psi_{Z}$ correspondingly; see Figure 1. All of the rotor's and ring's motions depend on each other since the bearing impedance forces couple them.

The changes of the momentum and angular momentum of the rotor (and wheels) per unit time are represented by the left-hand sides of (1) [29]. Due to the impedance forces of the bearings, the gravity forces, and the unbalance forces that act on the system, resultant forces and moments act on the 
system; these are expressed by the right-hand side of (1) with respect to its CM. The resulting impedance forces from the bearings to the rotor are $F_{Y, 1}$ and $F_{Y, 2}$ in the vertical direction and $F_{Z, 1}$ and $F_{Z, 2}$ in the horizontal direction; for bearing \#1 and bearing \#2 correspondingly, see Figure 1 .

$$
\begin{aligned}
M \ddot{y}_{\mathrm{CM}}= & F_{g, 1}+F_{Y, 1}+F_{g, 2}+F_{Y, 2}+\sum_{j=1}^{4} F_{u, y, j}, \\
M \ddot{z}_{\mathrm{CM}}= & F_{Z, 1}+F_{Z, 2}-\sum_{j=1}^{4} F_{u, z, j}, \\
J_{T} \ddot{\psi}_{Y}+J_{P} \Omega \dot{\psi}_{Z}= & F_{Y, 1} L_{1}-F_{Y, 2} L_{2}+F_{u, y, 1} U_{1}+F_{u, y, 2} U_{2} \\
& -F_{u, y, 3} U_{3}-F_{u, y, 4} U_{4}, \\
J_{T} \ddot{\psi}_{Z}-J_{P} \Omega \dot{\psi}_{Y}= & F_{Z, 1} L_{1}-F_{Z, 2} L_{2}-F_{u, z, 1} U_{1}-F_{u, z, 2} U_{2} \\
& +F_{u, z, 3} U_{3}+F_{u, z, 4} U_{4} .
\end{aligned}
$$

The system is characterized by 4 DoFs and the coordinates that can express the position of the rotor at any time moment include the pair of CM coordinates and two suitable angular coordinates. Due to the small clearances in bearings regarding the span between bearings $L_{1}+L_{2}$ (see Figure 1), the tilting angles $\psi_{Y}$ and $\psi_{Z}$ will be small for any rotor motion; thus, the system's nonlinearity is not assumed to be affected by the trigonometric functions of the fixed angular coordinates [16-18]. The system's unique source of nonlinearity is the fluid film bearing forces.

The four unbalance forces are applied on the planes defined at a distance $U_{j}$ from the plane of CM (see Figure 1); their definition is given in the following:

$$
\begin{aligned}
& F_{u, y, j}=u_{j} \Omega^{2} \cos \left(\frac{1}{2} \dot{\Omega} t^{2}\right), \\
& F_{u, z, j}=u_{j} \Omega^{2} \sin \left(\frac{1}{2} \dot{\Omega} t^{2}\right),
\end{aligned}
$$

$$
j=1,2,3,4 .
$$

The total gravity forces of the rotor and of the wheels transferred to the bearings are defined in (3) for each bearing; in all definitions subscript " 1 " implies bearing \#1 while subscript " 2 " implies bearing \#2:

$$
\begin{aligned}
& F_{g, 1}=-\frac{M g L_{2}}{\left(L_{1}+L_{2}\right)}, \\
& F_{g, 2}=-\frac{M g L_{1}}{\left(L_{1}+L_{2}\right)} .
\end{aligned}
$$

The formulas of (4) are used to express the motion of the $\mathrm{CM}$ and the tilting angles of the system with the four variables $y_{1}, y_{2}, z_{1}, z_{2}$ :

$$
\begin{aligned}
& y_{1}=y_{\mathrm{CM}}+L_{1} \psi_{Y}, \\
& y_{2}=y_{\mathrm{CM}}-L_{2} \psi_{Y}, \\
& z_{1}=z_{\mathrm{CM}}+L_{1} \psi_{Z}, \\
& z_{2}=z_{\mathrm{CM}}-L_{2} \psi_{Z}, \\
& \psi_{Y}=\frac{y_{1}-y_{2}}{L_{1}+L_{2}}, \\
& \psi_{Z}=\frac{z_{1}-z_{2}}{L_{1}+L_{2}} .
\end{aligned}
$$

After some math, the equations of motion expressed in (1) are expressed as in the following:

$$
\begin{aligned}
\ddot{y}_{1} & =\frac{L_{1}+L_{2}}{M L_{2}}\left(F_{g, 1}+F_{Y, 1}+F_{g, 2}+F_{Y, 2}+\sum_{j=1}^{4} F_{u, y, j}\right) \\
& -\frac{L_{1}}{L_{2}} \ddot{y}_{2}, \\
\ddot{z}_{1} & =\frac{L_{1}+L_{2}}{M L_{2}}\left(F_{Z, 1}+F_{Z, 2}-\sum_{j=1}^{4} F_{u, z, j}\right)-\frac{L_{1}}{L_{2}} \ddot{z}_{2}, \\
\ddot{y}_{2} & =-\frac{L_{1}+L_{2}}{J_{T}}\left(F_{Y, 1} L_{1}-F_{Y, 2} L_{2}+F_{u, y, 1} U_{1}+F_{u, y, 2} U_{2}\right. \\
& \left.-F_{u, y, 3} U_{3}-F_{u, y, 4} U_{4}\right)+\frac{J_{P} \Omega}{J_{T}}\left(\dot{y}_{1}-\dot{y}_{2}\right)+\ddot{z}_{1}, \\
\ddot{z}_{2} & =-\frac{L_{1}+L_{2}}{J_{T}}\left(F_{Z, 1} L_{1}-F_{Z, 2} L_{2}-F_{u, z, 1} U_{1}-F_{u, z, 2} U_{2}\right. \\
& \left.+F_{u, z, 3} U_{3}+F_{u, z, 4} U_{4}\right)-\frac{J_{P} \Omega}{J_{T}}\left(\dot{z}_{1}-\dot{z}_{2}\right)+\ddot{y}_{1} .
\end{aligned}
$$

Equations (7) and (8) show the ratio $J_{P} / J_{T}$. The value of this ratio specifies the number of critical speeds and, using linear analysis, we note that when $J_{P}>J_{T}$ the major critical speed is one while when $J_{P}<J_{T}$ the major critical speeds are two [29]. In this paper, nonlinear analysis will be performed.

The system of Figure 1 has two additional rigid bodies; these are the floating rings in the two bearings. Under the assumption of hydrodynamic lubrication, fluid film forces and moments act on the floating ring from the inner and the outer film of the lubricant. Resulting fluid film forces act on the ring from the outer film and are defined as $F_{Y, o}$ and $F_{Z, o}$ in the vertical and horizontal direction correspondingly; see Figure 2. In addition, resulting fluid film forces act from the inner film on the ring and are defined as $F_{Y, i}$ and $F_{Z, i}$ in the vertical and horizontal direction correspondingly; see Figure 2. Additionally, resulting torques from the inner and outer film act on the ring due to the rotation of the shaft and the ring. These are defined as $T_{i}$ and $T_{o}$ for inner and outer film correspondingly. The gravity forces are also incorporated 


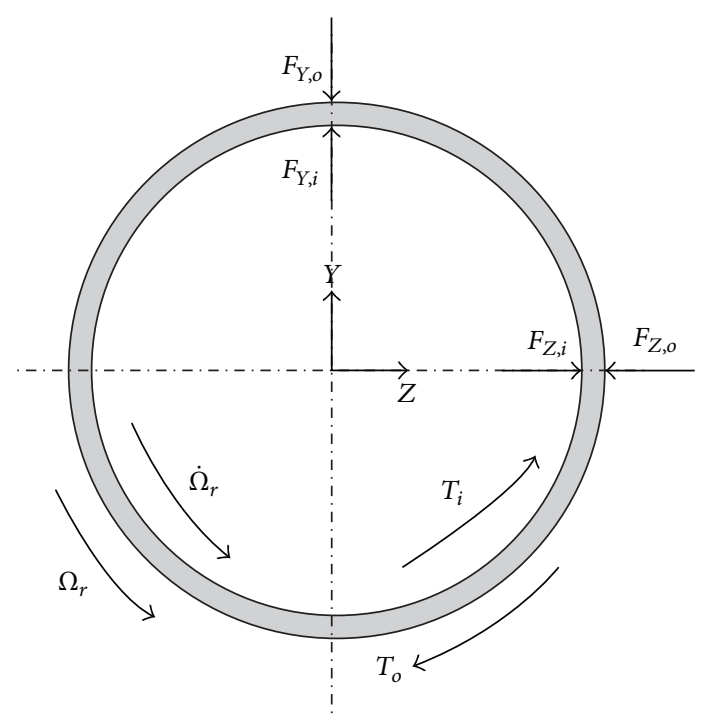

FIGURE 2: Representation of the forces and moments acting at the floating ring.

assumed to act on the vertical direction at the $\mathrm{CM}$ of each ring with magnitudes $m_{1} g$ and $m_{2} g$.

Under the action of the forces and moments from the fluid films to the rings, each ring (at bearing $\# 1$ and bearing \#2) obtains angular rotation of $\vartheta_{1}$ and $\vartheta_{2}$, rotating speed of $\Omega_{r, 1}=\dot{\vartheta}_{1}$ and $\Omega_{r, 2}=\dot{\vartheta}_{2}$, and rotational acceleration of $\dot{\Omega}_{r, 1}=\ddot{\vartheta}_{1}$ and $\dot{\Omega}_{r, 2}=\ddot{\vartheta}_{2}$, respectively. The motion of each ring is defined in the global coordinate system (see Figure 1) by the planar displacements of their CM, $y_{r, 1}$ and $y_{r, 2}$ in the vertical direction, and $z_{r, 1}$ and $z_{r, 2}$ in the horizontal direction for bearing $\# 1$ and bearing $\# 2$ correspondingly. Three independent coordinates are required for the definition of the motion of each ring at every discrete time moment: the two planar displacements and the angle of rotation. Therefore, 6 more DoFs are added for the simulation of the rotor-bearing system. Setting $I_{P, 1}$ and $I_{P, 2}$ as the mass moment of inertia of each ring with respect to its axis of rotation and $m_{1}$ and $m_{2}$ as the mass of each of them, the equations of motion are defined as in the following for the first and the second ring:

$$
\begin{aligned}
& m_{1} \ddot{y}_{r, 1}=-m_{1} g+F_{Y, o, 1}-F_{Y, i, 1}, \\
& m_{1} \ddot{z}_{r, 1}=F_{Z, o, 1}-F_{Z, i, 1}, \\
& m_{2} \ddot{y}_{r, 2}=-m_{2} g+F_{Y, o, 2}-F_{Y, i, 2}, \\
& m_{2} \ddot{z}_{r, 2}=F_{Z, o, 2}-F_{Z, i, 2}, \\
& I_{P, 1} \ddot{\vartheta}_{1}=T_{i, 1}-T_{o, 1}, \\
& I_{P, 2} \ddot{\vartheta}_{2}=T_{i, 2}-T_{o, 2} .
\end{aligned}
$$

Since the nonlinear fluid film bearing forces are evaluated through the dynamic motion of the floating rings, (5) to (9) present a 2nd-order nonlinear system. After the definition of each variable as in (10) the 2 nd-order $10 \times 10$ system of motion equations ((5) to (9)) is reduced to a 1st-order $20 \times 20$ system of motion equations as in (11):

$$
\begin{aligned}
& s(1)=y_{s, 1}, \\
& s(2)=\dot{y}_{s, 1} \text {, } \\
& s(3)=y_{s, 2} \text {, } \\
& s(4)=\dot{y}_{s, 2} \text {, } \\
& s(5)=z_{s, 1} \text {, } \\
& s(6)=\dot{z}_{s, 1} \text {, } \\
& s(7)=z_{s, 2} \text {, } \\
& s(8)=\dot{z}_{s, 2} \text {, } \\
& s(9)=y_{r, 1} \text {, } \\
& s(10)=\dot{y}_{r, 1} \text {, } \\
& s(11)=z_{r, 1} \text {, } \\
& s(12)=\dot{z}_{r, 1} \text {, } \\
& s(13)=\vartheta_{1} \text {, } \\
& s(14)=\dot{\vartheta}_{1} \text {, } \\
& s(15)=y_{r, 2}, \\
& s(16)=\dot{y}_{r, 2}, \\
& s(17)=z_{r, 2} \text {, } \\
& s(18)=\dot{z}_{r, 2} \text {, } \\
& s(19)=\vartheta_{2} \text {, } \\
& s(20)=\dot{\mathcal{\vartheta}}_{2} \text {. }
\end{aligned}
$$

The system $S(20 \times 20)$ can be solved with various methods of numerical integration of ODEs. According to the parameters of geometry and operation of the rotor-bearing system, the system $S(20 \times 20)$ can be less or more stiff. The Matlab solver "ode15s" $[21,22,30]$ integrates the system of differential equations $S(20 \times 20)$ in a discrete time domain after setting the initial conditions and the absolute and relevant error tolerance. It can integrate the system either with a fixed time interval size or without defined time interval size; in the second case, when the time step is not defined, the solver defines the time step size itself, according to the progress of the solution at certain discrete times. This makes the evaluation of the response during a system's run-up for virtual time of, for example, $10 \mathrm{~s}$, very fast (within minutes). During a run-up, the system experiences operational conditions that increase or decrease the nonlinearity of the fluid film forces and the suggested solver adopts its step in the progress of the solution. The "ode15s" is a variable order solver based on the numerical differentiation formulas (NDFs). Optionally, it uses the backward differentiation formulas (BDFs, also 
known as Gear's method) that are usually less efficient. It is suggested to try "ode15s" when an explicit Runge-Kutta fails or is very inefficient $[21,22]$. For problems such as the present, a Runge-Kutta does not fail but is judged to be inefficient due to the high evaluation time. The aspect of evaluation time will be discussed further later.

$S(20 \times 20)$

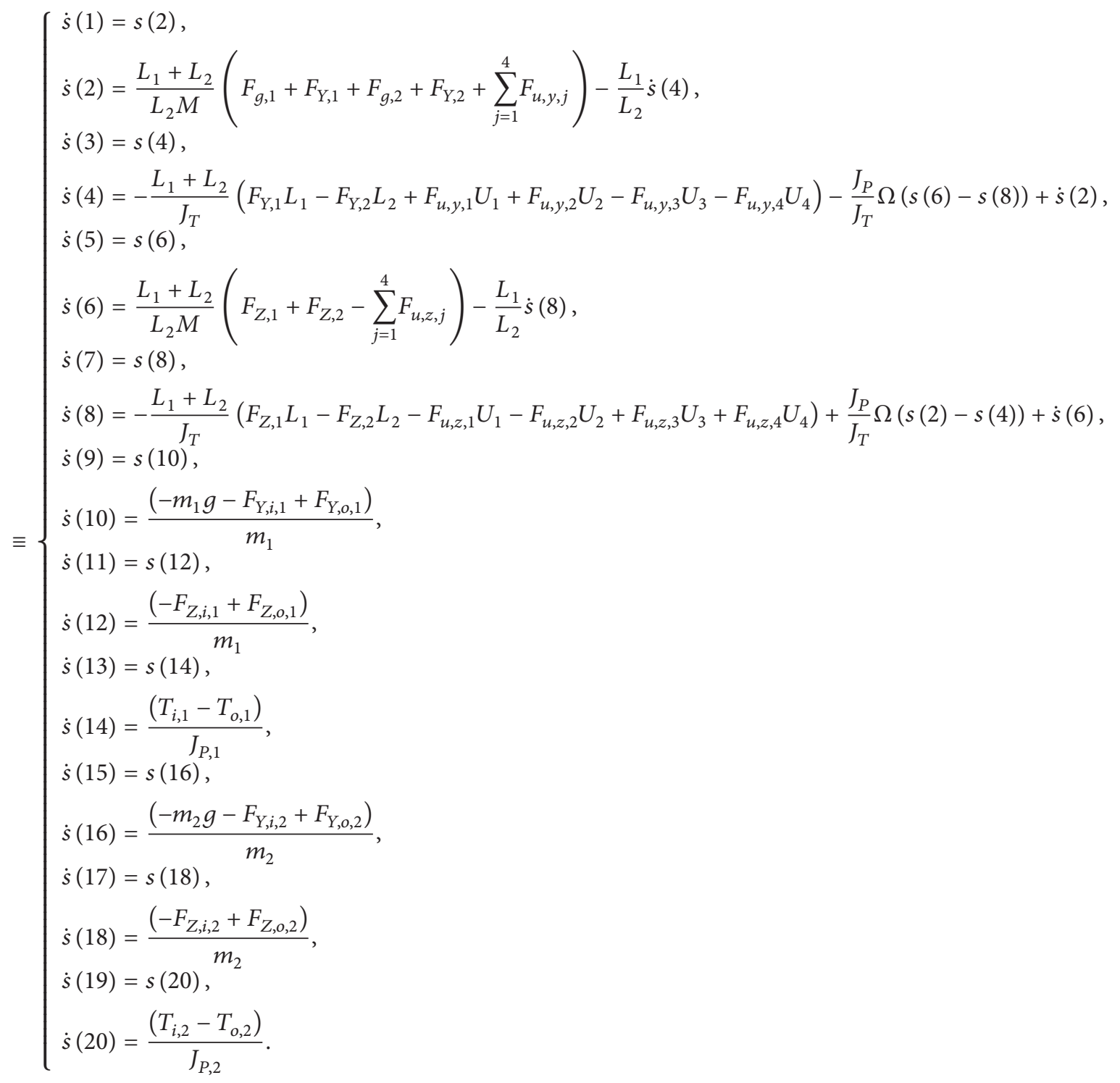

\section{The Analytical Model for the Finite-Length Floating Ring Bearing}

This section presents the theoretical model for the simulation of a finite-length floating ring bearing. The geometry of a plain cylindrical finite-length floating ring bearing is presented in Figure 3. The analysis considers a laminar, isothermal, isoviscous, incompressible flow of the lubricant that isolates the journal from the ring and the ring from the housing under the condition of purely hydrodynamic lubrication. The ring is assumed to be nondeformable, plain circular in both surfaces, and without holes; therefore no interference is assumed between the inner and the outer fluid film.

This section introduces the analytical solution of the Reynolds equation for both inner and outer film of the lubricant, considering dynamic conditions and finite bearing length. Two cases of solution will be defined: the exact analytical solution and the approximate analytical solution. The procedure of the analytical solution is common for both cases, exact and approximate, until a certain point, at which the mathematical procedure is separated to the two cases according to how one of the four Sturm-Liouville problems 

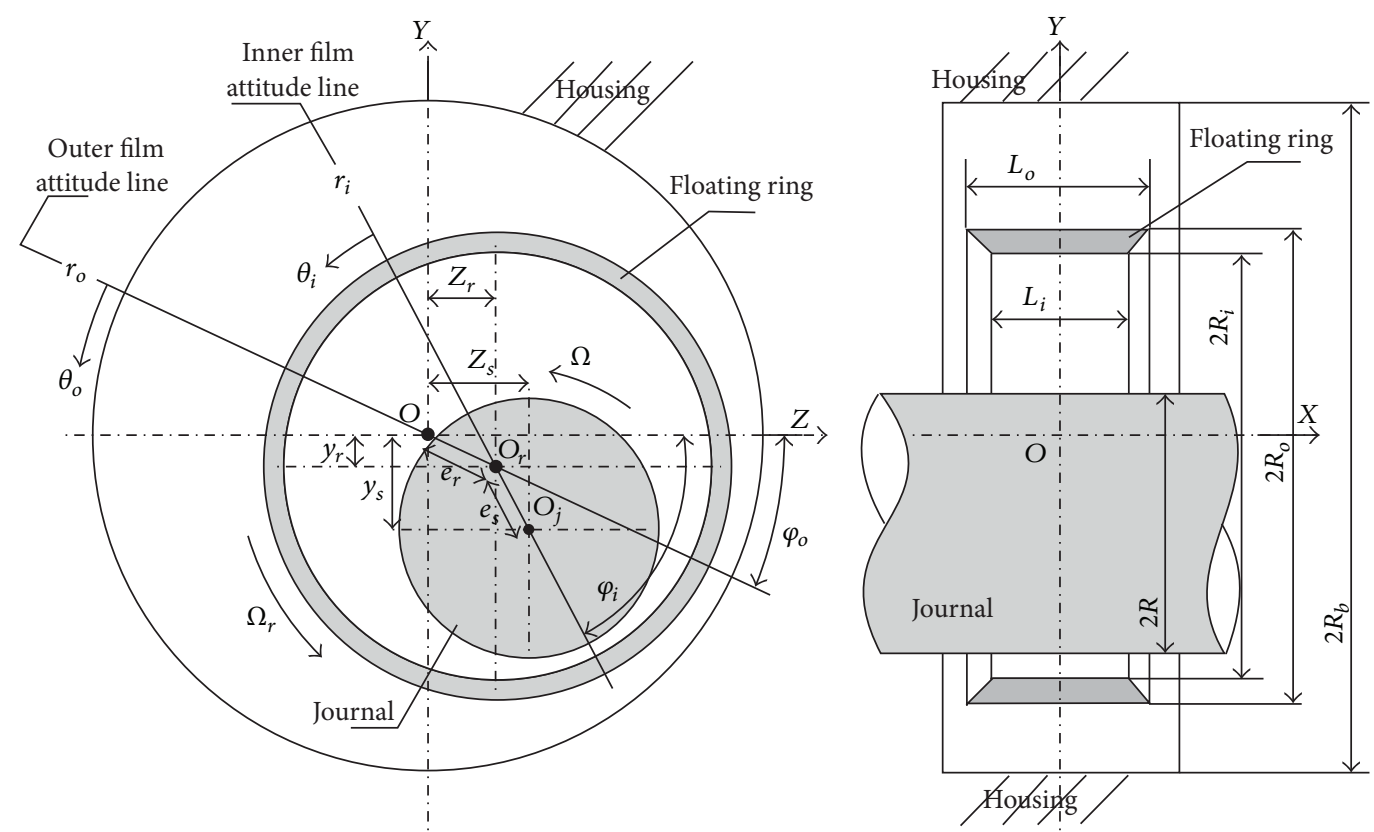

FIGURE 3: Definition of the geometry and parameters of operation used in the analysis of a plain cylindrical floating ring bearing.

(SLP) is confronted; this point is the end of the section and the further treatment has been presented in [26].

Before the treatment of the Reynolds solution, all parameters involved in the Reynolds equation have to be defined with respect to the state variables of the rotor-bearing system.

With reference to Figure 3, the inner eccentricity is defined as in (12) for both bearings; subscript " 1 " refers always to the left bearing (bearing \#1) and subscript " 2 " refers always to the right bearing (bearing \#2); see also Figure 1. The outer eccentricities are defined as in (13):

$$
\begin{aligned}
& e_{1}=\sqrt{\left(y_{1}-y_{r, 1}\right)^{2}+\left(z_{1}-z_{r, 1}\right)^{2}}, \\
& e_{2}=\sqrt{\left(y_{2}-y_{r, 2}\right)^{2}+\left(z_{2}-z_{r, 2}\right)^{2}}, \\
& e_{r, 1}=\sqrt{y_{r, 1}^{2}+z_{r, 1}^{2}}, \\
& e_{r, 2}=\sqrt{y_{r, 2}^{2}+z_{r, 2}^{2}} .
\end{aligned}
$$

By defining the radial clearances as in (14), the eccentricity ratios are defined for the inner films as in (15) and for the outer films as in (16):

$$
\begin{aligned}
c_{i} & =R_{i}-R, \\
c_{o} & =R_{b}-R_{o}, \\
\varepsilon_{i, 1} & =\frac{e_{1}}{c_{i, 1}}, \\
\varepsilon_{i, 2} & =\frac{e_{2}}{c_{i, 2}}, \\
\varepsilon_{o, 1} & =\frac{e_{r, 1}}{c_{o, 1}}, \\
\varepsilon_{o, 2} & =\frac{e_{r, 2}}{c_{o, 2}} .
\end{aligned}
$$

The problem is considered to be dynamic and the journal and the ring are assumed to execute any planar trajectories inside the clearance area of each film; the inner eccentricity rate of change in bearing \#1 is defined as in (17) and in bearing $\# 2$ as in (18):

$\dot{e}_{1}$

$$
=\frac{\left(\left(y_{s, 1}-y_{r, 1}\right)\left(\dot{y}_{s, 1}-\dot{y}_{r, 1}\right)+\left(z_{s, 1}-z_{r, 1}\right)\left(\dot{z}_{s, 1}-\dot{z}_{r, 1}\right)\right)}{e_{1}},
$$

$\dot{e}_{2}$

$$
=\frac{\left(\left(y_{s, 2}-y_{r, 2}\right)\left(\dot{y}_{s, 2}-\dot{y}_{r, 2}\right)+\left(z_{s, 2}-z_{r, 2}\right)\left(\dot{z}_{s, 2}-\dot{z}_{r, 2}\right)\right)}{e_{2}} .
$$

The following equation defines the corresponding rate of change of the outer eccentricities in the bearings:

$$
\begin{aligned}
& \dot{e}_{r, 1}=\frac{\left(y_{r, 1} \dot{y}_{r, 1}+z_{r, 1} \dot{z}_{r, 1}\right)}{e_{r, 1}}, \\
& \dot{e}_{r, 2}=\frac{\left(y_{r, 2} \dot{y}_{r, 2}+z_{r, 2} \dot{z}_{r, 2}\right)}{e_{r, 2}} .
\end{aligned}
$$

The rates of change of the attitude angles (see Figure 3 ) of the inner films are defined in (20) for bearing \#1 and in (21) 
for bearing \#2. The rate of change of the attitude angles of the outer films is defined in (22) for both bearings:

$$
\begin{aligned}
& \dot{\varphi}_{i, 1} \\
& =\frac{\left(\left(z_{s, 1}-z_{r, 1}\right)\left(\dot{y}_{s, 1}-\dot{y}_{r, 1}\right)-\left(y_{s, 1}-y_{r, 1}\right)\left(\dot{z}_{s, 1}-\dot{z}_{r, 1}\right)\right)}{e_{1}^{2}}, \\
& \dot{\varphi}_{i, 2} \\
& =\frac{\left(\left(y_{s, 2}-y_{r, 2}\right)\left(\dot{y}_{s, 2}-\dot{y}_{r, 2}\right)+\left(z_{s, 2}-z_{r, 2}\right)\left(\dot{z}_{s, 2}-\dot{z}_{r, 2}\right)\right)}{e_{2}^{2}}, \\
& \dot{\varphi}_{o, 1}=\frac{\left(z_{r, 1} \dot{y}_{r, 1}-y_{r, 1} \dot{z}_{r, 1}\right)}{e_{r, 1}^{2}}, \\
& \dot{\varphi}_{o, 2}=\frac{\left(z_{r, 2} \dot{y}_{r, 2}-y_{r, 2} \dot{z}_{r, 2}\right)}{e_{r, 2}^{2}} .
\end{aligned}
$$

The rotating speed of the rings is defined in (23) and then the effective rotating speed on each of the four fluid films can be defined as in (24) for the inner films and as in (25) for the outer films. Equation (23) makes a reference to the system's equations (see (11)):

$$
\begin{aligned}
\Omega_{r, 1} & =\dot{\vartheta}_{1}=s(14), \\
\Omega_{r, 2} & =\dot{\vartheta}_{2}=s(20), \\
\Omega_{\mathrm{eff}, i, 1} & =\Omega+\Omega_{r, 1}-2 \dot{\varphi}_{i, 1}, \\
\Omega_{\mathrm{eff}, i, 2} & =\Omega+\Omega_{r, 2}-2 \dot{\varphi}_{i, 2}, \\
\Omega_{\mathrm{eff}, o, 1} & =\Omega_{r, 1}-2 \dot{\varphi}_{o, 1}, \\
\Omega_{\mathrm{eff}, o, 2} & =\Omega_{r, 2}-2 \dot{\varphi}_{o, 2} .
\end{aligned}
$$

The analysis incorporates the analytical solution of the Reynolds equation using global geometric and physical parameters of the fluid film that are defined below and in the Nomenclature. In a fluid film consisting of two finite-length cylindrical surfaces (journal and bearing), let $c$ be the radial clearance, $e$ the eccentricity, $\mu$ the dynamic viscosity of the lubricant, $\Omega_{\text {eff }}$ the effective rotational speed in the fluid film, $L_{b}$ the length of the fluid film, and $R$ the smallest of the radius of the surfaces that form each of the fluid films; then, the fluid film thickness with respect to the angular coordinate $\theta$ of the film of consideration is defined as in (26) and the developed pressure distribution $P(x, \theta)$ of the lubricant through the coordinates $\theta$ and $x$ (see Figure 3 ) will be given from the analytical solution of the Reynolds equation written as in (27):

$$
\begin{aligned}
& h=c+e \cos (\theta), \\
& \frac{h^{3}}{\mu R^{2}} \frac{\partial P^{2}(x, \theta)}{\partial \theta^{2}}-\frac{3 e h^{2} \sin (\theta)}{\mu R^{2}} \frac{\partial P(x, \theta)}{\partial \theta} \\
& \quad+\frac{h^{3}}{\mu} \frac{\partial P^{2}(x, \theta)}{\partial x^{2}}=12 \dot{e} \cos (\theta)-6 e \Omega_{\mathrm{eff}} \sin (\theta) .
\end{aligned}
$$

Referring to the recent achievement of the analytical solution of the Reynolds equation for the plain cylindrical journal bearing $[23,24]$, the floating ring bearing [26, 27], and the three-lobe journal bearing [25], this section will only present the milestones of the analytical procedure; this will make the solution understandable and compact. The procedure concerns one fluid film and the definitions of the last paragraph above will be used.

The solution of the Reynolds equation $P(x, \theta)$ is expressed as the sum of a particular solution of the Reynolds, $u(x, \theta)$, and of the solution of the homogenous Reynolds, $g(x, \theta)$, as in (28) [23-27]. The homogenous Reynolds is expressed as in (27) where the right-hand part of the equation is set to zero.

$$
\begin{aligned}
& P(x, \theta)=u(x, \theta)+g(x, \theta), \\
& u(x, \theta)=\Psi(x)+\Phi(\theta) \\
& g(x, \theta)=m(x) f(\theta)
\end{aligned}
$$

The particular solution will be given using the additive separation of variables and the homogenous solution will be given using the multiplicative separation of variables. Thus, the particular solution $u(x, \theta)$ is expressed as the sum of the single variable functions $\Psi(x)$ and $\Phi(\theta)$ while the homogenous solution $g(x, \theta)$ is expressed as the product of the single variable functions $m(x)$ and $f(\theta)$; see (29).

Substituting $P(x, \theta)$ with $u(x, \theta)$ in the Reynolds equation (26) and with $g(x, \theta)$ in the homogenous Reynolds equation, the following are formulated:

$$
\begin{aligned}
& \frac{h^{3}}{\mu R^{2}} \frac{\partial u^{2}(x, \theta)}{\partial \theta^{2}}-\frac{3 e h^{2} \sin (\theta)}{\mu R^{2}} \frac{\partial u(x, \theta)}{\partial \theta} \\
& +\frac{h^{3}}{\mu} \frac{\partial u^{2}(x, \theta)}{\partial x^{2}}=12 \dot{e} \cos (\theta)-6 e \Omega_{\mathrm{eff}} \sin (\theta), \\
& \frac{h^{3}}{\mu R^{2}} \frac{\partial g^{2}(x, \theta)}{\partial \theta^{2}}-\frac{3 e h^{2} \sin (\theta)}{\mu R^{2}} \frac{\partial g(x, \theta)}{\partial \theta} \\
& +\frac{h^{3}}{\mu} \frac{\partial g^{2}(x, \theta)}{\partial x^{2}}=0 .
\end{aligned}
$$

The use of the formulas defined in (29) in the corresponding equations (30) leads to separation of variables and four 2nd-order ODEs are formed as in (31) to (34). The boundary conditions for each of them are also presented in the corresponding equations, forming four Sturm-Liouville problems (SLPs). The set of boundary conditions of the Reynolds equation in this paper is the Gumbel/half Sommerfeld boundary condition. This is implemented with an ambient or zero pressure at the start of the oil film (position of highest fluid film thickness; see Figure 3) and an ambient or zero pressure at the end of the oil film (position of lowest fluid film thickness; see Figure 3), when the journal is assumed to rotate around a fixed point (static conditions). In the case of dynamic conditions (journal lateral motion), the ambient/zero pressure is applied at the beginning of the oil film if the journal's radial velocity (direction " $r$ "; see Figure 3 ) is not so high/negative so as to provoke cavitation on the 
side of the minimum fluid film thickness (rare case). The negative pressure in all cases is not taken into account in the integration that will yield the fluid film forces. The "general" boundary conditions are distributed to the four SL problems in the way shown in (31)-(34). A detailed explanation for the definition of the boundary conditions can be found in $[23,26]$.

$$
\begin{aligned}
& \frac{1}{R^{2}} \frac{d^{2} \Phi(\theta)}{d \theta^{2}}+\frac{3}{R^{2}} \frac{1}{h} \frac{d h}{d \theta} \frac{d \Phi(\theta)}{d \theta} \\
& \quad-\left(\frac{6 \mu \Omega_{\mathrm{eff}}}{h^{3}} \frac{d h}{d \theta}+\frac{12 \mu}{h^{3}} \frac{d h}{d t}\right)=0, \\
& \Phi(0)=\Phi(2 \pi)=0 \\
& \frac{d^{2} \Psi(x)}{d x^{2}}=0, \\
& \Psi\left(-\frac{L_{b}}{2}\right)=\Psi\left(\frac{L_{b}}{2}\right)=0, \\
& \frac{d^{2} m(x)}{d x^{2}}=k^{2} m(x), \\
& m\left(-\frac{L_{b}}{2}\right)=m\left(\frac{L_{b}}{2}\right)=1,
\end{aligned}
$$

$$
k \in R^{*}
$$

$$
\begin{aligned}
& \frac{d^{2} f(\theta)}{d \theta^{2}}+\frac{3}{h} \frac{d h}{d \theta} \frac{d f(\theta)}{d \theta}+k^{2} R^{2} f(\theta)=0, \\
& f(0)=f(2 \pi)=0
\end{aligned}
$$

$$
k \in R^{*} .
$$

The direct analytical solution of the SLP in (31) is given in (35) [26], while the solution of $\Psi(x)$ is chosen to be the trivial (36); see also [25] for a short explanation. The direct analytical solution of the SLP in (33) is given in (37) which defines its eigenfunctions [26]. The SLP of (34) has no direct analytical solution and this is due to the trigonometric function of the fluid film thickness $h$ [31].

$\Phi(\theta)$

$$
\begin{gathered}
=\frac{6 R^{2} \mu \sin (\theta / 2)}{h^{2}}\left(\frac{2 \mathrm{e} \Omega_{\mathrm{eff}} \cos (\theta / 2)(2 c+e \cos (\theta))}{2 c^{2}+e^{2}}\right. \\
\left.+\frac{2 \dot{e}(2 c+e+e \cos (\theta)) \sin (\theta / 2)}{(c+e)^{2}}\right), \\
\Psi(x) \equiv 0, \\
m_{j}(x)=\frac{\mathrm{e}^{j L_{b} /(4 R)}}{1+\mathrm{e}^{j L_{b} /(2 R)}}\left(\mathrm{e}^{j x /(2 R)}+\mathrm{e}^{-j x /(2 R)}\right), \\
\quad j=1,2, \ldots .
\end{gathered}
$$

Supposing that the eigenfunctions $f_{j}(\theta)$ of the SLP for $f(\theta)$ can be evaluated, the homogenous Reynolds solution $g(x, \theta)$ would be defined as in (38) with $\delta_{j}$ being constants of the general solution for $g(x, \theta)$ that will be defined in the following sections according to the solution for $f(\theta)$. It is proved that only a number of $K=7 \sim 10$ eigenmodes are enough for the accurate evaluation of the homogenous solution [26].

$$
g(x, \theta)=\sum_{j=1}^{K}\left(\delta_{j} m_{j}(x) f_{j}(\theta)\right), \quad \delta_{j} \in R, K \in Z^{*} .
$$

At this point, the analysis is separated into two cases, the approximate analytical solution for $f(\theta)$ and thus Reynolds's approximate solution and the exact analytical solution for $f(\theta)$ and thus Reynolds's exact solution [26].

\section{Transient Response Analysis}

The results consider two high-speed rotor-bearing systems taken from applications of automotive turbochargers. The first geometric configuration is described in main dimensions in Figure 4(a) and the second in Figure 4(b). The first system, "system \#1," is lighter, is smaller in all dimensions, and operates at higher speed than the second system, "system \#2." These two geometric configurations are assumed to be near the lower and upper limit on the range of dimensions and speeds in which the automotive turbochargers of passenger cars are designed to operate nowadays. Thus, systems \#1 and \#2 are considered to define a wide range of dimensions/weights/speeds of turbochargers. Regarding the system size, thermodynamic and structural dynamic issues set the operating speed range. In such systems, only a slight change in dimensions and especially in the dimensions of the bearing clearances, bearing diameter, and length can yield very different results regarding the instability margins, amplitude of instability response and can set the configuration out of order. Implementing these two systems in the analysis, this study intends to cover two very different cases of automotive turbocharger rotor dynamic simulation, in order to investigate the applicability of the exact analytical solution for the floating ring bearing.

System \#1 has its ending speed at $240000 \mathrm{RPM}(4000 \mathrm{~Hz})$ while the ending speed of system \#2 is set at 171900 RPM $(2865 \mathrm{~Hz})$. In real operating conditions, the ending speed margins are expected to be similar. Additionally, system \#1 is noted for an asymmetry in its bearing properties while the bearings of system $\# 2$ have equal geometrical and physical properties. The detailed geometrical and physical configuration of system \#1 is presented in Table 1 and of system \#2 in Table 2.

The floating ring bearings of the two systems are simulated under the solutions presented in the previous sections and described briefly in Table 3.

A transient run-up with initial conditions almost to zero is performed for both systems with a constant rotational acceleration $\dot{\Omega}$ that results in linear speed variation and thus linear driving frequency variation from zero to the ending speed $\Omega_{\max }$ defined for each system in Tables 2 and 3 correspondingly. The results consist of time-series of displacement, bearing eccentricity, ring speed ratio, journal 


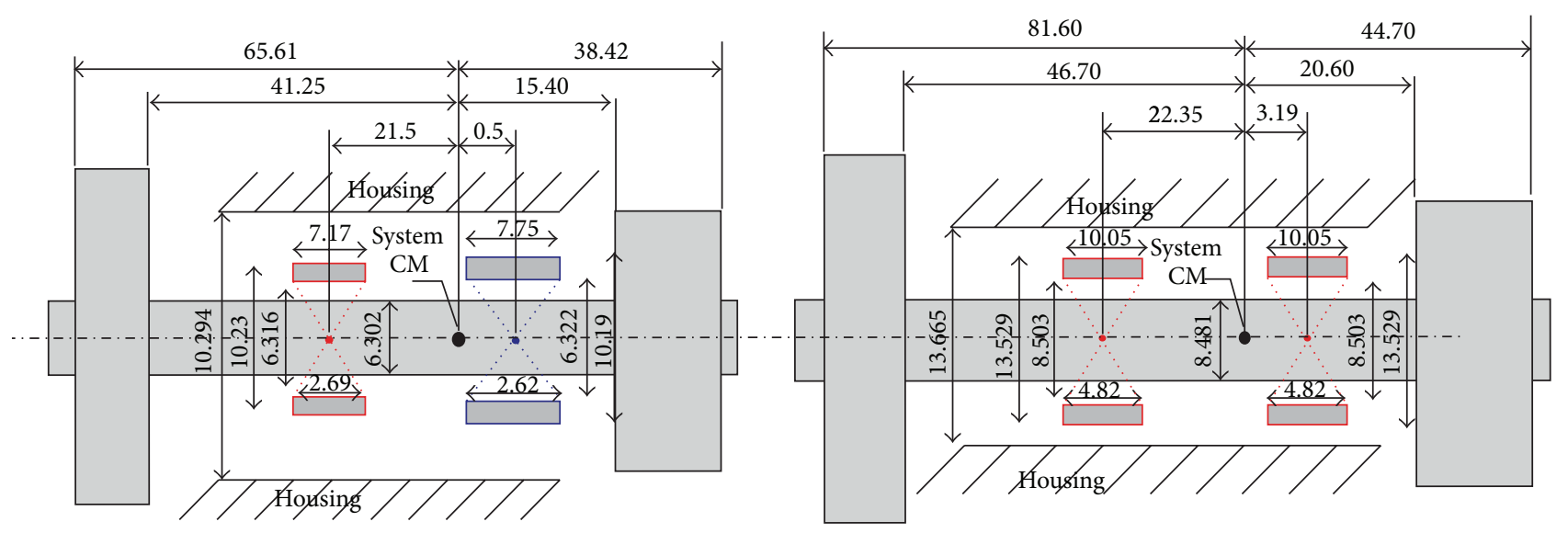

(a)

(b)

Figure 4: Representation and basic dimensions of the simulated systems; (a) system \#1 and (b) system \#2, in mm.

TABLE 1: Definition of the geometric and physical properties of system \#1 (higher speed).

\begin{tabular}{|c|c|c|}
\hline Rotor & Bearing \#1 & Bearing \#2 \\
\hline$M=0.119 \mathrm{~kg}$ & $c_{i, 1}=6.5 e-6 \mathrm{~m}$ & $c_{i, 2}=14 e-6 \mathrm{~m}$ \\
\hline$J_{P}=10.60 e-6 \mathrm{kgm}^{2}$ & $c_{o, 1}=32 e-6 \mathrm{~m}$ & $c_{o, 2}=38.5 e-6 \mathrm{~m}$ \\
\hline$J_{T}=153.56 e-6 \mathrm{kgm}^{2}$ & $m_{1}=0.00265 \mathrm{~kg}$ & $m_{2}=0.00260 \mathrm{~kg}$ \\
\hline$L_{1}=0.0215 \mathrm{~m}$ & $R_{1}=0.0031515 \mathrm{~m}$ & $R_{2}=0.003147 \mathrm{~m}$ \\
\hline$L_{2}=0.0005 \mathrm{~m}$ & $R_{i, 1}=0.003158 \mathrm{~m}$ & $R_{i, 2}=0.003161 \mathrm{~m}$ \\
\hline$\dot{\Omega}=1675.51 \mathrm{rad} / \mathrm{s}^{2}$ & $R_{o, 1}=0.005115 \mathrm{~m}$ & $R_{o, 2}=0.005095 \mathrm{~m}$ \\
\hline$\Omega_{\max }=25132.74 \mathrm{rad} / \mathrm{s}(240000 \mathrm{RPM})$ & $L_{i, 1}=0.00269 \mathrm{~m}$ & $L_{i, 2}=0.00262 \mathrm{~m}$ \\
\hline$t_{\max }=15 \mathrm{~s}$ & $L_{o, 1}=0.00717 \mathrm{~m}$ & $L_{o, 2}=0.00775 \mathrm{~m}$ \\
\hline$U_{1}=0.06561 \mathrm{~m}$ & $I_{P, 1}=5.207 e-6 \mathrm{kgm}^{2}$ & $I_{P, 2}=5.205 e-6 \mathrm{kgm}^{2}$ \\
\hline$U_{2}=0.04125 \mathrm{~m}$ & $T_{i, 1}=160^{\circ} \mathrm{C}$ & $T_{i, 2}=160^{\circ} \mathrm{C}$ \\
\hline$U_{3}=0.01540 \mathrm{~m}$ & $T_{o, 1}=150^{\circ} \mathrm{C}$ & $T_{o, 2}=150^{\circ} \mathrm{C}$ \\
\hline$U_{4}=0.03842 \mathrm{~m}$ & $\mu_{i, 1}=0.0024 \mathrm{Pas}$ & $\mu_{i, 2}=0.0024 \mathrm{Pas}$ \\
\hline$u_{i}=5 e-8 \mathrm{kgm}, i=1,2,3,4$ & $\mu_{o, 1}=0.0028 \mathrm{Pas}$ & $\mu_{o, 2}=0.0028 \mathrm{Pas}$ \\
\hline
\end{tabular}

TABLE 2: Definition of the geometric and physical properties of system \#2 (lower speed).

\begin{tabular}{|c|c|c|}
\hline Rotor & Bearing \#1 & Bearing \#2 \\
\hline$M=0.246 \mathrm{~kg}$ & $c_{i, 1}=34 e-6 \mathrm{~m}$ & $c_{i, 2}=34 e-6 \mathrm{~m}$ \\
\hline$J_{P}=36.47 e-6 \mathrm{kgm}^{2}$ & $c_{o, 1}=68 e-6 \mathrm{~m}$ & $c_{o, 2}=68 e-6 \mathrm{~m}$ \\
\hline$J_{T}=524.26 e-6 \mathrm{kgm}^{2}$ & $m_{1}=0.00605 \mathrm{~kg}$ & $m_{2}=0.00605 \mathrm{~kg}$ \\
\hline$L_{1}=0.02235 \mathrm{~m}$ & $R_{1}=0.0042405 \mathrm{~m}$ & $R_{2}=0.0042405 \mathrm{~m}$ \\
\hline$L_{2}=0.00319 \mathrm{~m}$ & $R_{i, 1}=0.0042515 \mathrm{~m}$ & $R_{i, 2}=0.0042515 \mathrm{~m}$ \\
\hline$\dot{\Omega}=1800.13 \mathrm{rad} / \mathrm{s}^{2}$ & $R_{o, 1}=0.0067645 \mathrm{~m}$ & $R_{o, 2}=0.0067645 \mathrm{~m}$ \\
\hline$\Omega_{\max }=18001.33 \mathrm{rad} / \mathrm{s}(171900 \mathrm{RPM})$ & $L_{i, 1}=0.00482 \mathrm{~m}$ & $L_{i, 2}=0.00482 \mathrm{~m}$ \\
\hline$t_{\max }=10 \mathrm{~s}$ & $L_{o, 1}=0.01005 \mathrm{~m}$ & $L_{o, 2}=0.01005 \mathrm{~m}$ \\
\hline$U_{1}=0.08160 \mathrm{~m}$ & $I_{P, 1}=0.197 e-6 \mathrm{kgm}^{2}$ & $I_{P, 2}=0.197 e-6 \mathrm{kgm}^{2}$ \\
\hline$U_{2}=0.04670 \mathrm{~m}$ & $T_{i, 1}=150^{\circ} \mathrm{C}$ & $T_{i, 2}=150^{\circ} \mathrm{C}$ \\
\hline$U_{3}=0.02060 \mathrm{~m}$ & $T_{o, 1}=140^{\circ} \mathrm{C}$ & $T_{o, 2}=140^{\circ} \mathrm{C}$ \\
\hline$U_{4}=0.04470 \mathrm{~m}$ & $\mu_{i, 1}=0.0028 \mathrm{Pas}$ & $\mu_{i, 2}=0.0028 \mathrm{Pas}$ \\
\hline$u_{i}=1 e-7 \mathrm{kgm}, i=1,2,3,4$ & $\mu_{o, 1}=0.0033$ Pas & $\mu_{o, 2}=0.0033 \mathrm{Pas}$ \\
\hline
\end{tabular}


TABLE 3: Definition/description of the cases of simulation for the high-speed rotor-bearing systems.

\begin{tabular}{lccl}
\hline Case & Rotor model & Bearing model & Bearing solution \\
\hline$\# 1$ & Rigid & Finite length bearing & Exact analytical solution \\
$\# 2$ & Rigid & Finite length bearing & Approximate analytical solution, Bessel eigfs. \\
$\# 3$ & Rigid & Short bearing approximation & Approximate analytical solution \\
$\# 4$ & Rigid & Finite length bearing & Numerical solution (Finite Difference Method) \\
\hline
\end{tabular}

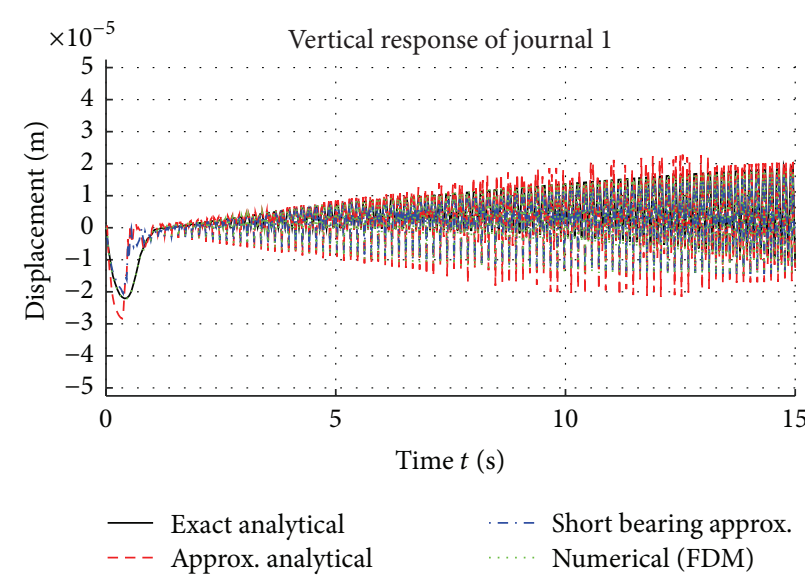

(a)

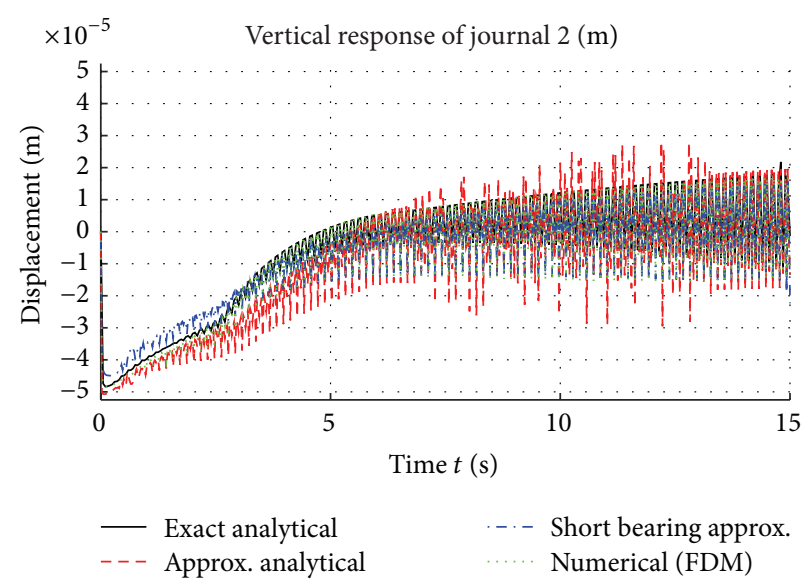

(b)

FIGURE 5: System \#1: vertical displacement of the journal as a function of time for the different cases of simulation; (a) bearing \#1 and (b) bearing $\# 2$.

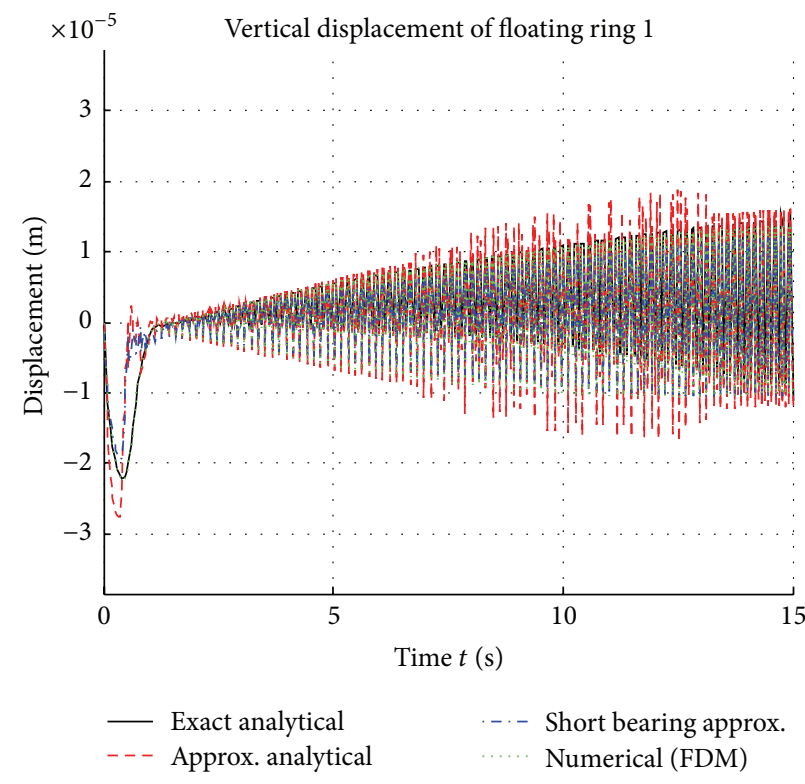

(a)

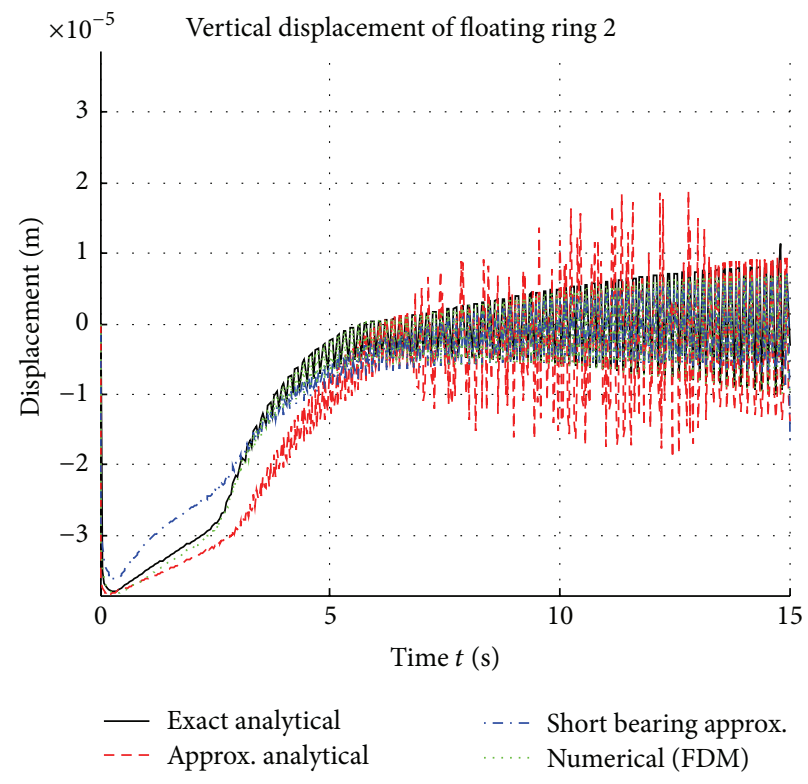

(b)

FIGURE 6: System \#1: vertical displacement of the floating ring as a function of time for the different cases of simulation; (a) bearing \#1 and (b) bearing \#2.

and ring trajectories, and time-frequency analysis of rotor response for both simulated systems.

4.1. High-Speed System \#1. This section analyses the evaluated response of the system shown in Figure 4(a). The displacement of the rotor at the location of the journals and the displacement of the floating rings are shown in Figures 5 and 6 correspondingly for a transient run-up of the system as defined in the beginning of Section 4 for four cases of bearing simulation: exact analytical, approximate analytical, short bearing approximation, and numerical solution using FDM. 
A similar progress of the displacement over time is noted for both journals and rings. The journal and ring response evaluated through the exact analytical solution are almost equal to those evaluated through the numerical solution using FDM regarding amplitude and instability thresholds. The short bearing approximation yields lower response amplitude of journals and rings through the entire run-up time; this can be clearly noted in Figures 14 and 15 and no postprocessing is required for precise amplitude calculation. An short instability is presented at the very early time of run-up, when exact analytical solution and short bearing approximation are used; this can be clearly shown later in the time-frequency analysis (see Figures 10(a) and 10(c)). Regarding the lower speeds during the run-up (time up to $5 \mathrm{~s}$ ) where the journals and the rings execute trajectories around an equilibrium position, we note a divergence regarding the equilibrium locus evaluated through approximate analytical and the other three solutions. It should be noted that, as shown in Section 3, the pressure distribution through approximate analytical solution converges better with other solutions when eccentricities are low (0.1-0.3). The major difference among the solutions is noted regarding an instability threshold produced only from the approximate analytical solution at about between $7 \mathrm{~s}$ and $13 \mathrm{~s}$ of run-up. The pressure distribution is assumed to be the reason and further discussion is added below.

The eccentricities presented in the bearing are plotted in Figure 7, where we note a different progress of the dynamic behaviour of the system among the different solutions. Figure 7 clearly shows that the exact analytical and the numerical solution using FDM are in very good agreement. A minimal divergence is noted between them only when the speed gets higher (after $10 \mathrm{~s}$ ). The short bearing approximation yields slightly lower eccentricities through almost the entire duration of run-up. The event of total instability seems to start developing very close to the ending speed and is produced only by the short bearing approximation and the numerical solution using FDM. It should be noted here that total instability will be produced also by the exact analytical solution and the approximate analytical solution, probably at a higher speed. However, the simulated ending speed is already high enough for the specific application.

The ring speed is a parameter of major importance for the development of instabilities. The ring speed, together with the rotor speed and the rotor's whirling frequency, defines the effective speed in the fluid film; see (24) and (25). The ring speed is plotted in Figure 8 for both bearings. The progress of the ring speed among the different cases of simulation can be assumed to be in correspondence to the instabilities noted, but in the current example this is not as clear as in system \#2, analysed in the next section. Figure 8 shows that the bearing solutions yield almost the same ring speed in ring 1 and for the first $5 \mathrm{~s}$; afterwards, as the speed gets higher, the ring speed evaluated through the approximate analytical solution starts to diverge in value and in progress as well having the tendency to increase more rapidly in ring 2 compared to the other solutions.

The journal orbits are extracted from the time-series of displacement during run-up for the specific rotating speeds of $\Omega=50 \mathrm{kRPM}, \Omega=100 \mathrm{kRPM}, \Omega=150 \mathrm{kRPM}$, and $\Omega=200 \mathrm{kRPM}$ and for the duration of one driving period at each speed; see Figure 9. Figure 9 shows that, in general, the orbits declare linear stable motion. Only at speed of $\Omega=$ $150 \mathrm{kRPM}$ and $\Omega=200 \mathrm{kRPM}$ the orbits evaluated through the approximate analytical solution present a nonclosing form that implies an additional whirling to the synchronous; in journal 2 this is noted more clearly. The specific speeds of $\Omega=150 \mathrm{kRPM}$ and $\Omega=200 \mathrm{kRPM}$ exist in the range where the instability occurs at the case of approximate analytical solution (see Figures 14-16). Further comments on the instability will be added after the time-frequency analysis.

The time-series presented in Figure 5 are decomposed in time and frequency domain through Short Time Fourier Transform (STFT). This decomposition is always performed during turbosystem design and the spectrograms are assumed to be of major importance for deciding whether to accept the design. The instabilities developed produce different sounds regarding their frequency and the avoidance or suppression of these instabilities and the corresponding sounds are of major importance for acceptance of the design. However, the presence of instabilities can also be influenced by other parameters, such as the vibrations of the foundation, which in current applications is a motor. Figure 10 shows that the exact analytical solution (Figure 10(a)) and the short bearing approximation (Figure 10(c)) develop only an instability at a very low rotating speed, meaning 5000 RPM-10000 RPM for a turbosystem. The amplitude of the component corresponding to this instability, called sub-1, can be low or high; in this application, it presents a relatively low amplitude. The numerical solution using FDM (see Figure 10(d)) does not produce this instability. The approximate analytical solution using Bessel's functions produces the sub-1 component and as the speed gets higher it also produces the sub-3 component that is related to the third instability usually noted in such systems. Again, the amplitude of sub-3 component can be low or high and plays a decisive role in acceptance of the design. In the current application, the amplitude of the components due to instability is low compared to that of the component of frequency synchronous to the driving frequency, noted as "syn." After these notifications, the current bearing geometry is assumed to compose a very efficient design and this can be achieved with confidence even through a short bearing approximation regarding the bearing simulation. Although the length-to-diameter ratio of the fluid film is $L_{i, 1} /\left(2 R_{i, 1}\right)=0.42, L_{i, 2} /\left(2 R_{i, 2}\right)=0.41, L_{o, 1} /\left(2 R_{o, 1}\right)=$ 0.70 , and $L_{o, 2} /\left(2 R_{o, 2}\right)=0.76$ for the corresponding fluid film, the short bearing approximation can be judged to be sufficient for the simulation of the bearings. This is not always the case, as will be shown later. In the current system, the exact analytical solution contributes only to the more precise vibration amplitude that is in agreement with the numerical solution using FDM and in evaluation of the response that will be discussed at the end of Section 4 .

4.2. High-Speed System \#2. This section analyses the evaluated response of the system shown in Figure 4(b). The displacement of the rotor at the location of the journals and 


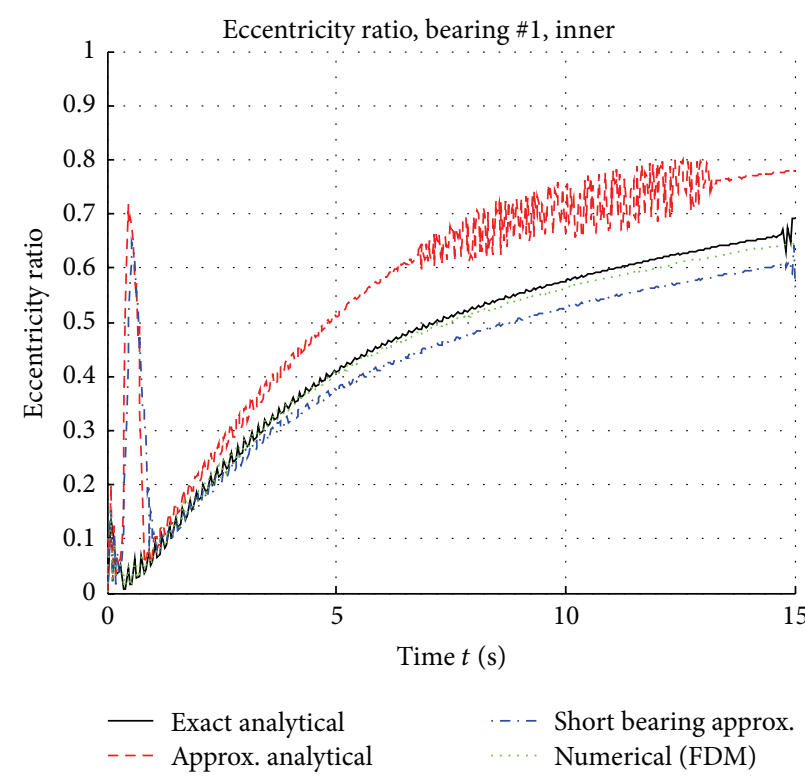

(a)

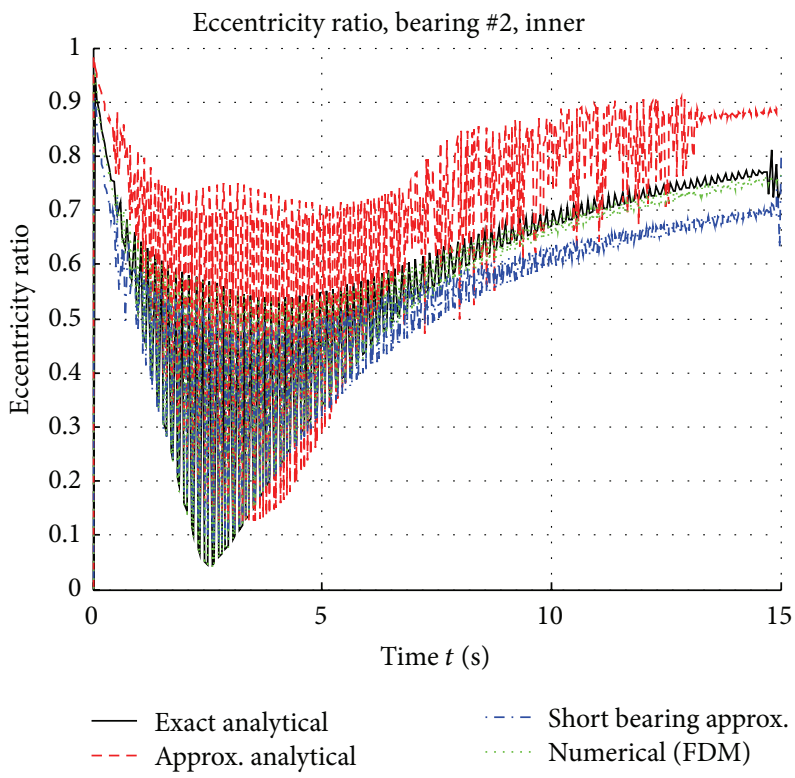

(c)

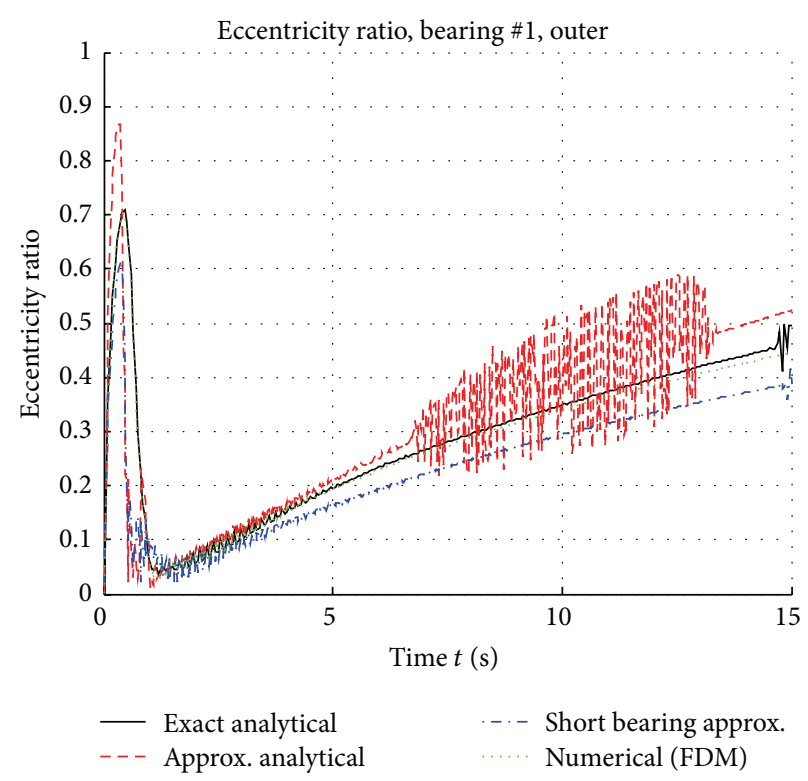

(b)

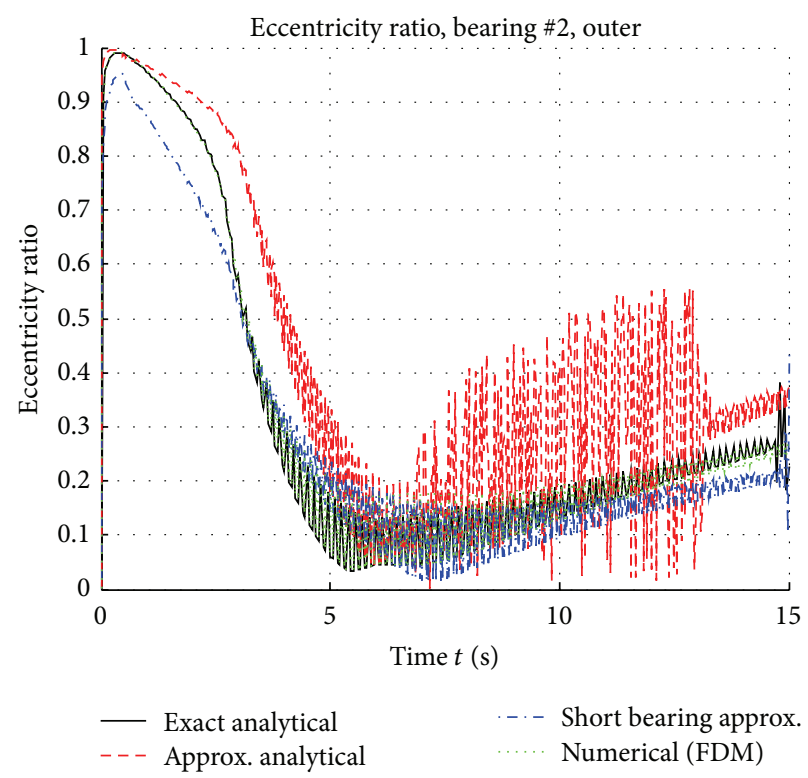

(d)

FIGURE 7: System \#1: eccentricity ratio as a function of time for the different cases of simulation; (a) bearing \#1, inner film, (b) bearing \#1, outer film, (c) bearing \#2, inner film, and (d) bearing \#2, outer film.

the displacement of the floating rings are shown in Figures 11 and 12 correspondingly for a transient run-up of the system as defined in the beginning of Section 4 for three cases of bearing simulation: exact analytical solution, approximate analytical solution, and short bearing approximation. This section does not present the results considering numerical solution using FDM because they were found again in almost absolute agreement with those from the exact analytical solution, as happened in the simulation of system \#1 in Section 4.1. Considering the results within the numerical solution, only the time-frequency analysis is presented at the end of this section, where a slight difference is presented regarding developed instability.

A similar progress of displacement over time is noted for both journals and rings among the approximate analytical solution and the short bearing approximation. However, the amplitude is slightly different with the approximate analytical solution to produce higher amplitude of response near radial clearance. Furthermore, the approximate analytical solution produces instability at lower speed than the short bearing approximation. The exact analytical solution produces a different response than that of approximate analytical solution 


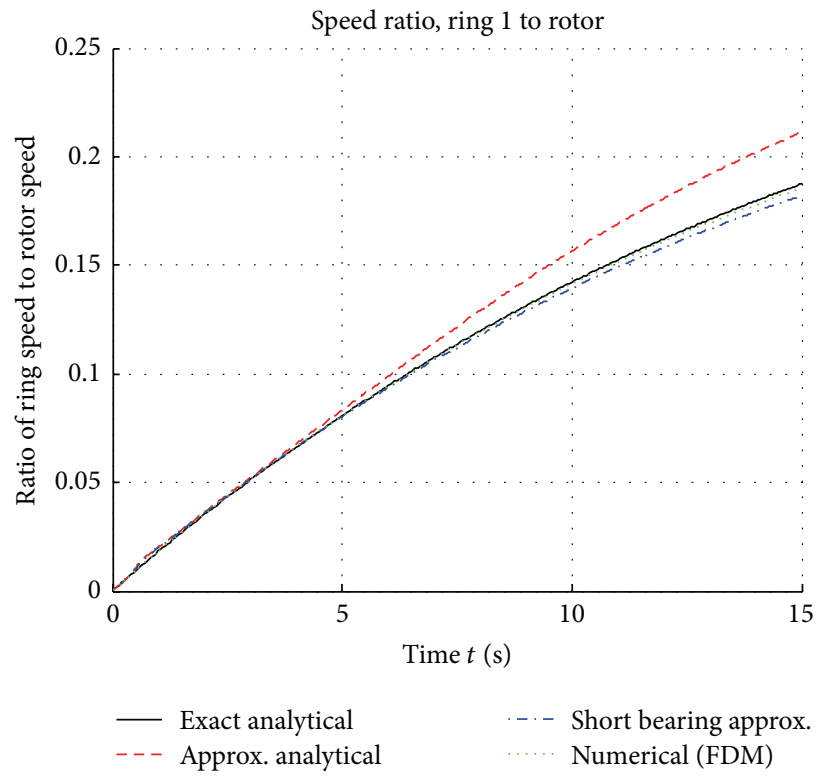

(a)

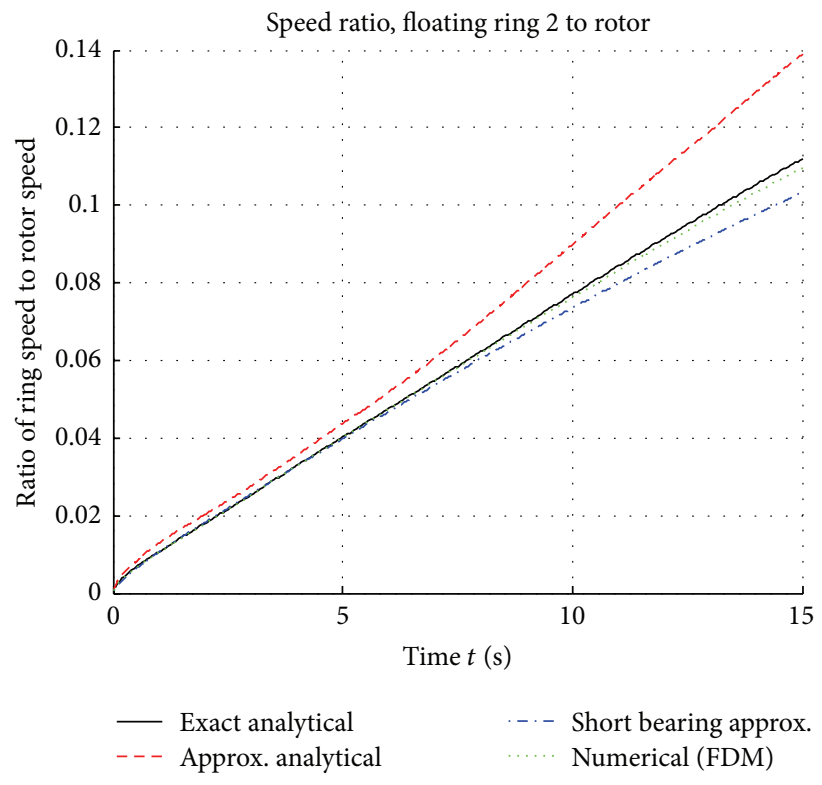

(b)

FIGURE 8: System \#1: ratio of the speed of the floating ring to the rotor's speed as a function of time for the different cases of simulation; (a) bearing \#1 and (b) bearing \#2.

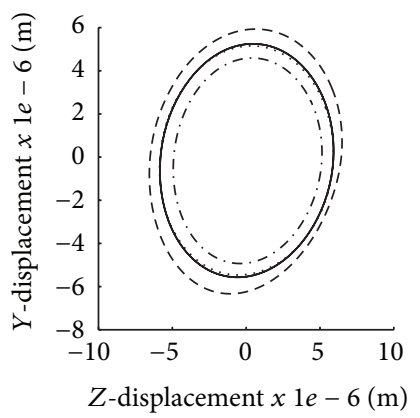

(a)

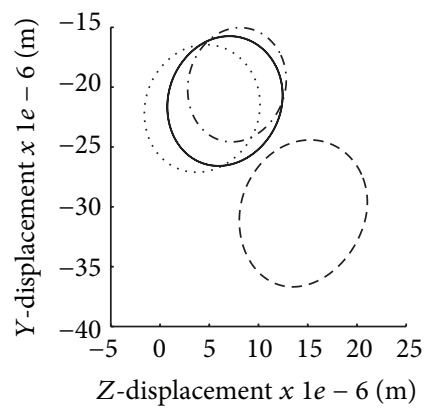

(e)

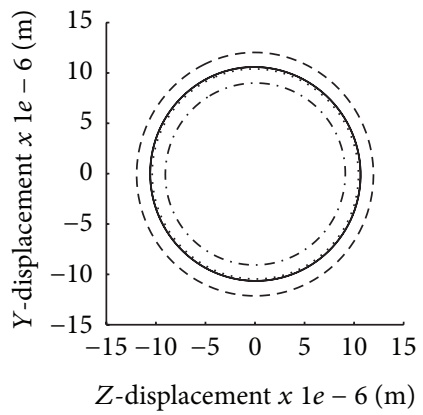

(b)

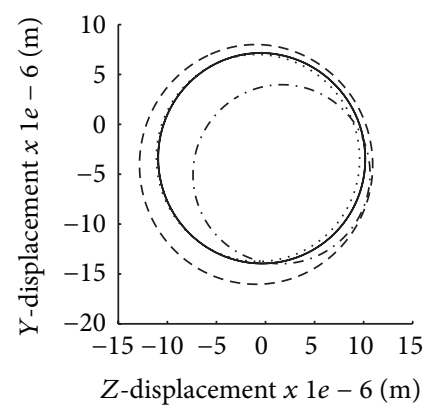

(f)

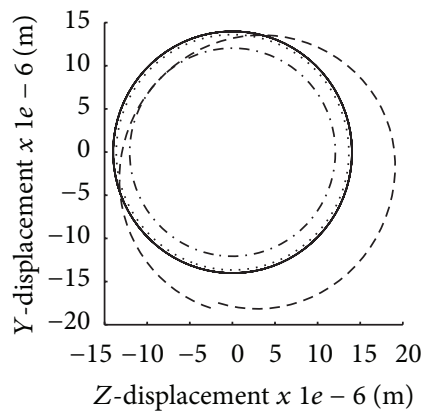

(c)

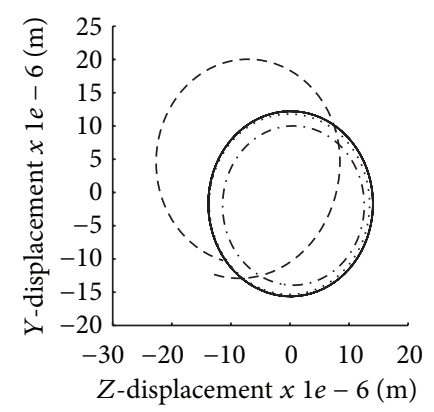

(g)

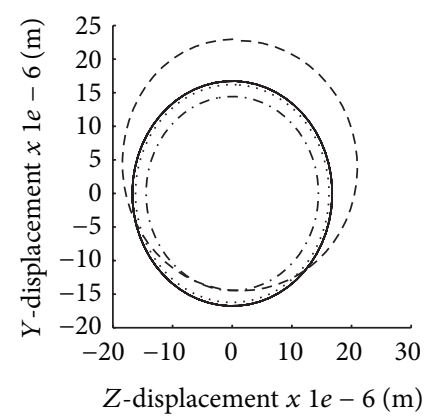

(d)

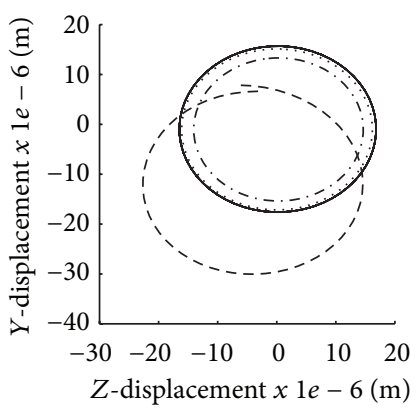

(h)

FIGURE 9: System \#1: orbits of the journal at bearing \#1 (a, b, c, d) and bearing \#2 (e, f, g, h) for the different cases of simulation at (a) and (e): $\Omega=50 \mathrm{kRPM}$; (b) and (f): $\Omega=100 \mathrm{kRPM}$; (c) and (g): $\Omega=150 \mathrm{kRPM}$; and (d) and (h) $\Omega=200 \mathrm{kRPM}$. Solid line: exact analytical solution (Case \#1), dashed line: approximate analytical solution (Case \#2), dash-dotted line: short bearing approximation (Case \#3), and dotted line: numerical solution (FDM) (Case \#4). See also Table 3 for the definition of the cases. 


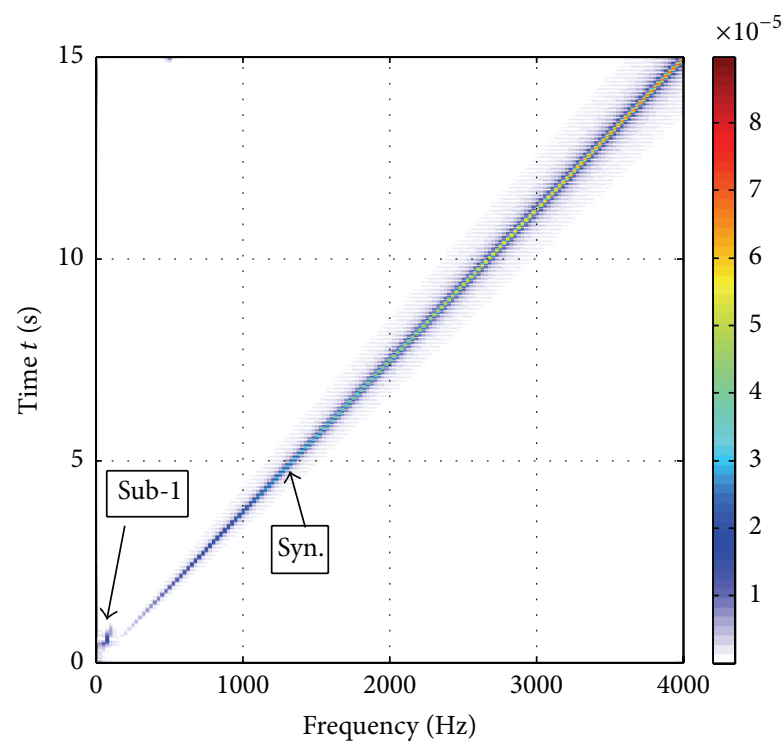

(a)

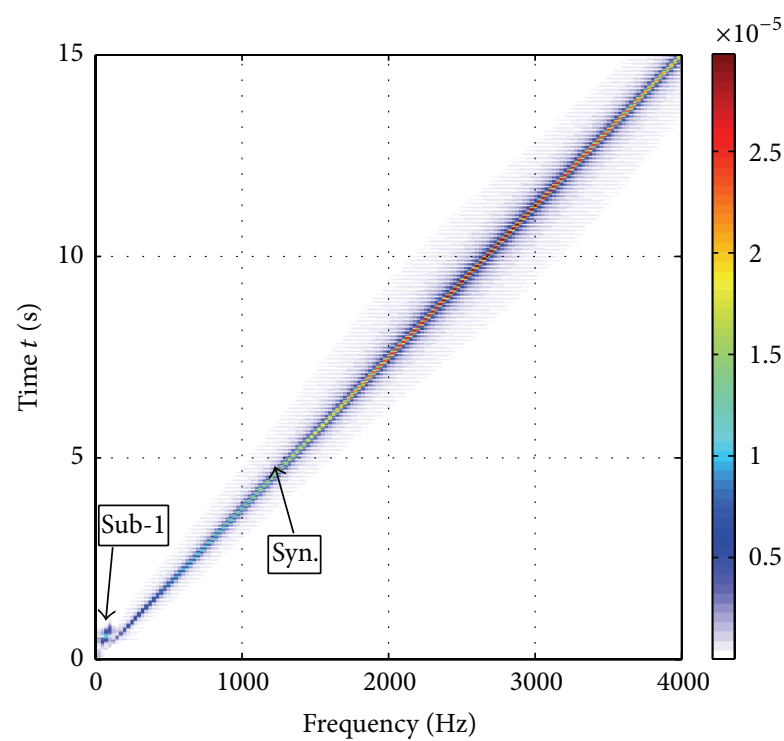

(c)

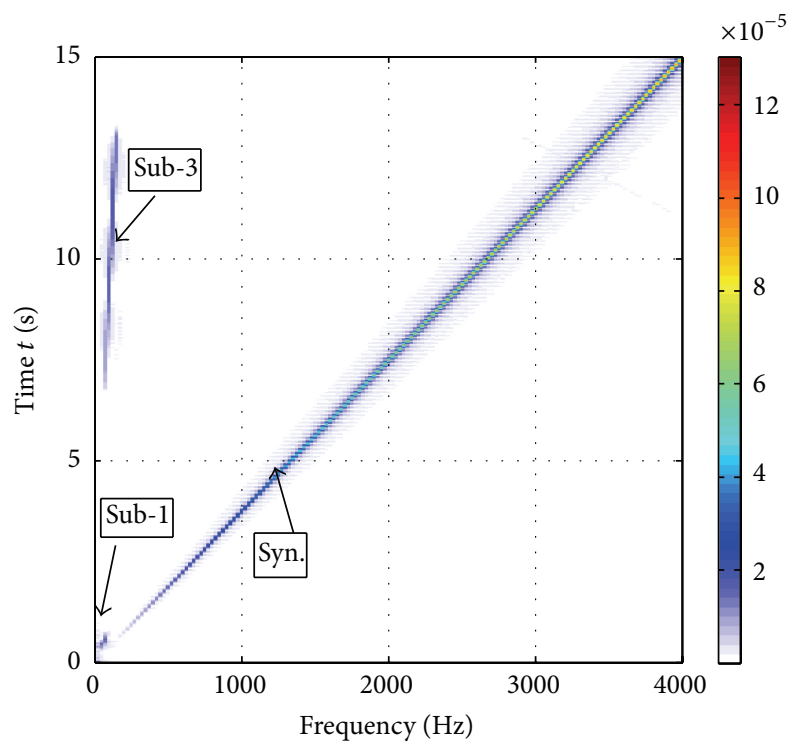

(b)

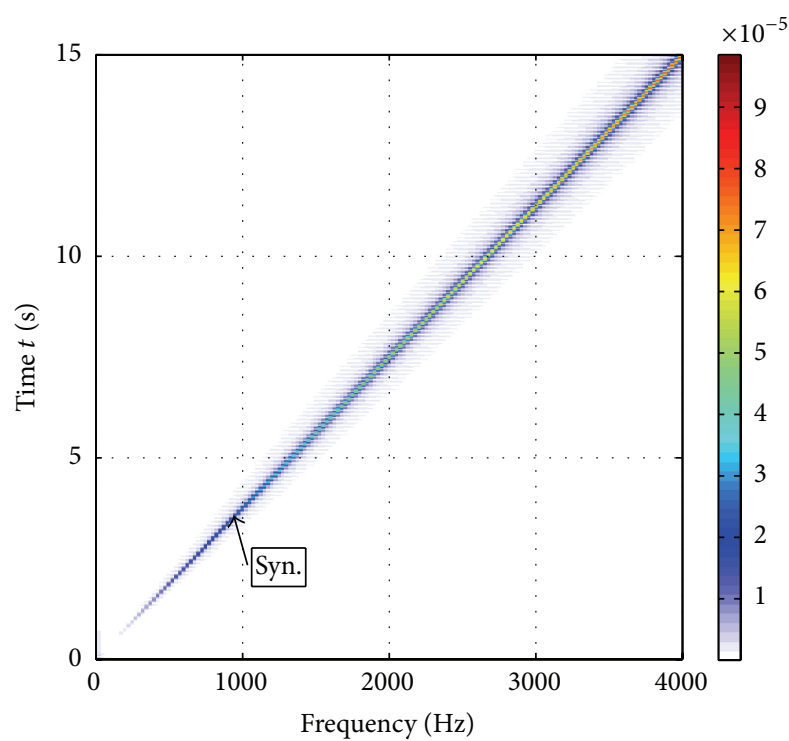

(d)

FIGURE 10: System \#1: time-frequency decomposition using STFT of the transient response of the journal of bearing \#1 for the simulation of (a) Case \#1, (b) Case \#2, (c) Case \#3, and (d) Case \#4.

and short bearing approximation, regarding progress, amplitude, and instability threshold. The journal and ring response evaluated through the exact analytical solution are almost equal to those evaluated through the numerical solution using FDM regarding amplitude and instability thresholds but are not presented in the charts in order to leave room for the notification of the other two solutions. The short bearing approximation yields lower response amplitude of journals and rings through the entire time of run-up; this can be clearly noted in Figures 11 and 12 and no postprocessing is required for the precise amplitude calculation; the same observation was also made in system \#1. The response using the exact analytical solution is of relatively low amplitude up to the time of $4.5 \mathrm{~s}$ and then it gradually increases and reaches its maximum near the radial clearance at about the ending speed.

A long instability of relatively constant amplitude near the radial clearance is presented at the early time of runup, at about $2 \mathrm{~s}$ using approximate analytical solution and at about $3 \mathrm{~s}$ using short bearing approximation; this can be clearly shown later in the time-frequency analysis (see Figures 16(a) and 16(c)). Regarding the lower speeds during the run-up (time up to $1.5 \mathrm{~s}$ ) where the journals and the rings execute trajectories around an equilibrium position, we note a divergence regarding the equilibrium locus evaluated through approximate analytical and the other two solutions. 


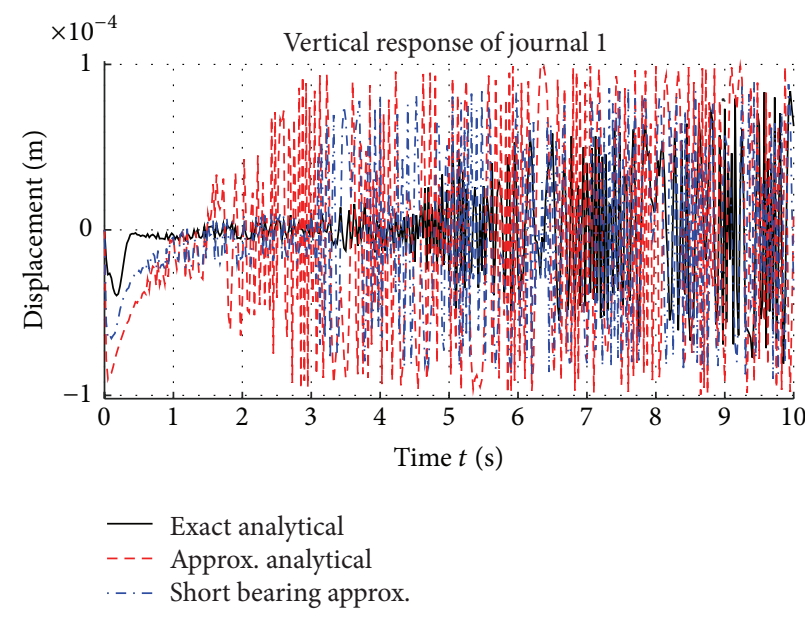

(a)

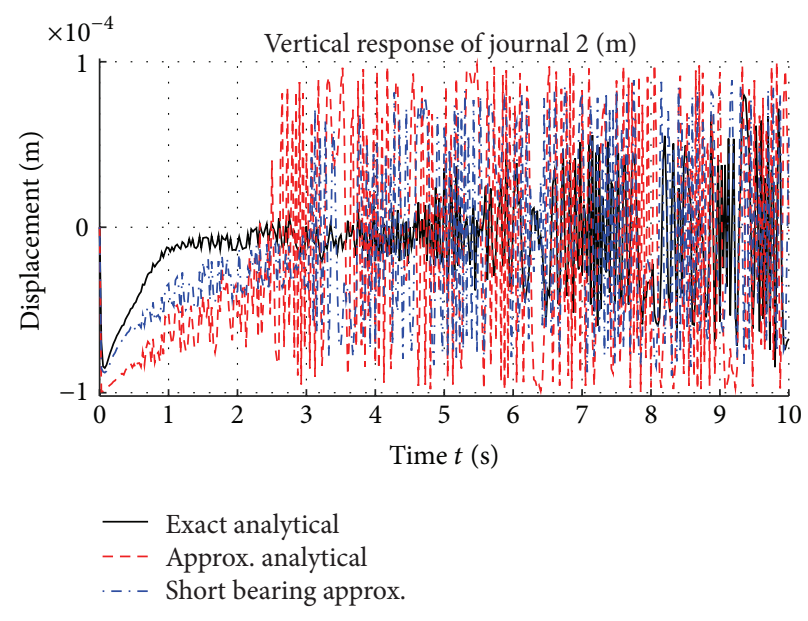

(b)

FIGURE 11: System \#2: vertical displacement of the journal as a function of time for the different cases of simulation; (a) bearing \#1 and (b) bearing $\# 2$.

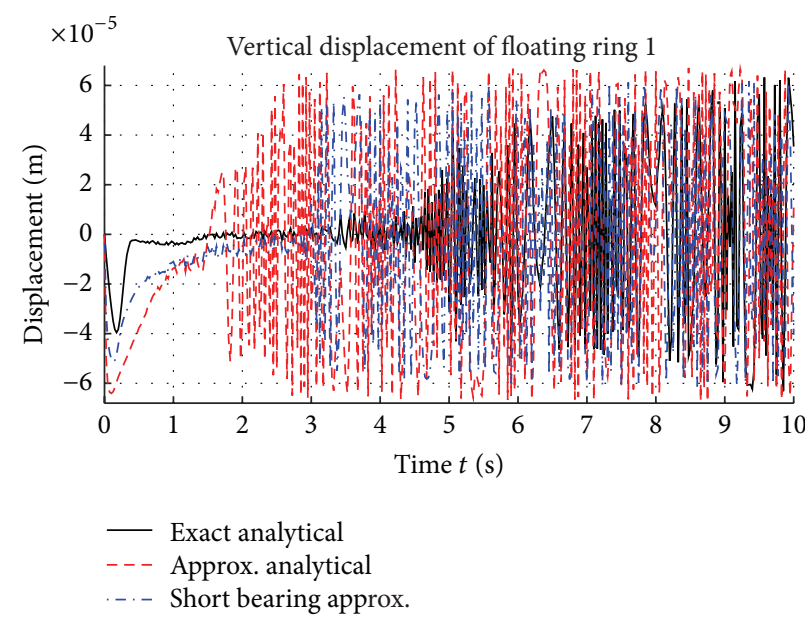

(a)

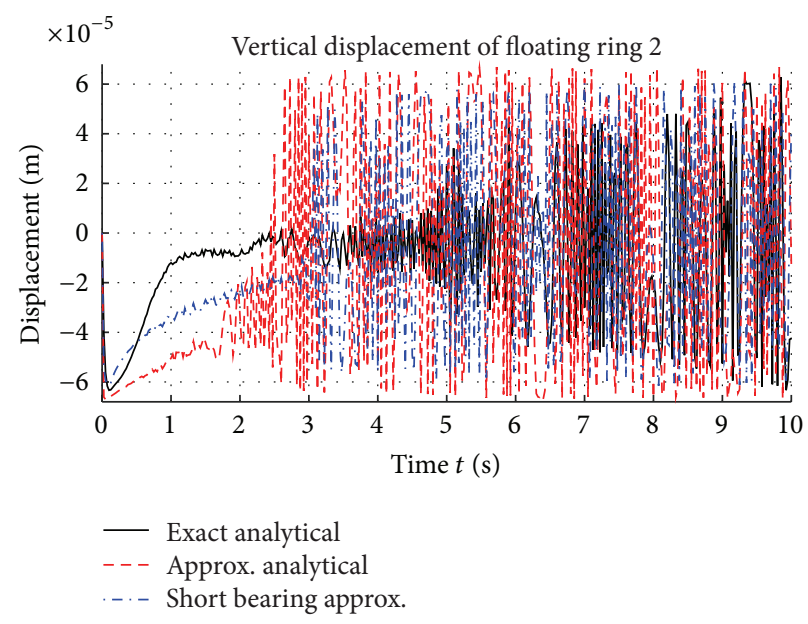

(b)

FIGURE 12: System \#2: vertical displacement of the floating ring as a function of time for the different cases of simulation; (a) bearing \#1 and (b) bearing \#2.

It should be noted that, as shown in Section 3, the pressure distribution through approximate analytical solution converges better with the other solutions when eccentricities are low (0.1-0.3). The major difference among the solutions is noted regarding the instability threshold and amplitude of the corresponding component. Again, as in system \#1, the pressure distribution is assumed to be the reason and further discussion is added below.

The eccentricities presented at the bearing are plotted in Figure 13, which clearly shows how the exact analytical solution becomes different from the short bearing approximation and the approximate analytical solution. The short bearing approximation yields slightly lower eccentricities through almost the entire duration of run-up compared to that evaluated through the approximate analytical solution. However, they have a very similar quality in their progress.
The exact analytical solution yields eccentricities that are of much lower amplitude compared to the other two solutions and the progress is different, indicating a potential source of instability at about $5 \mathrm{~s}$ in the outer fluid films and at about $8.5 \mathrm{~s}$ in the inner fluid films.

The plot of the ring speed in Figure 14 indicates in correspondence to Figure 13 the thresholds of instability. Figure 14 shows that the ring speed is "locked" to a constant ratio as the instability occurs under the approximate analytical solution and the short bearing approximation. On the contrary, the exact analytical solution yields a different progress of ring speed especially at higher speeds; however, at lower speeds the progress and the values of ring speed are found in quite good agreement among the bearing solutions.

The journal orbits are extracted from the time-series of displacement during run-up for the specific rotating speeds 

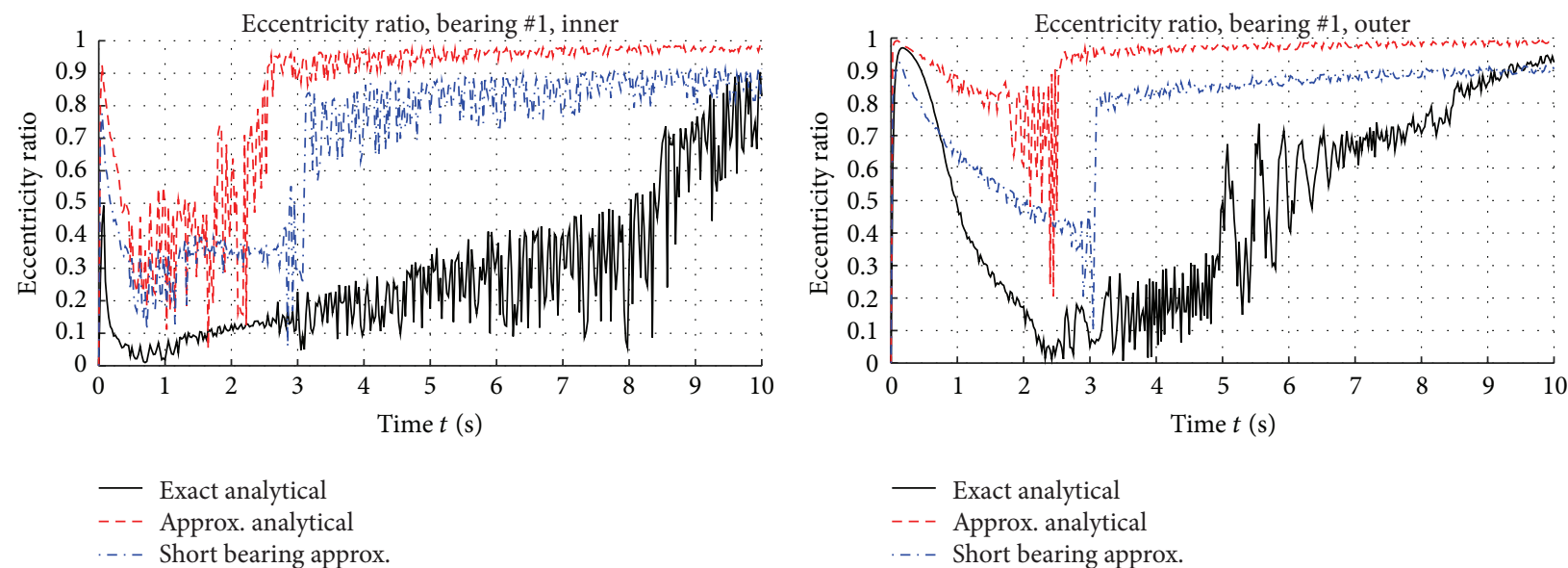

- Exact analytical

- - Approx. analytical

-..- Short bearing approx.

(a)
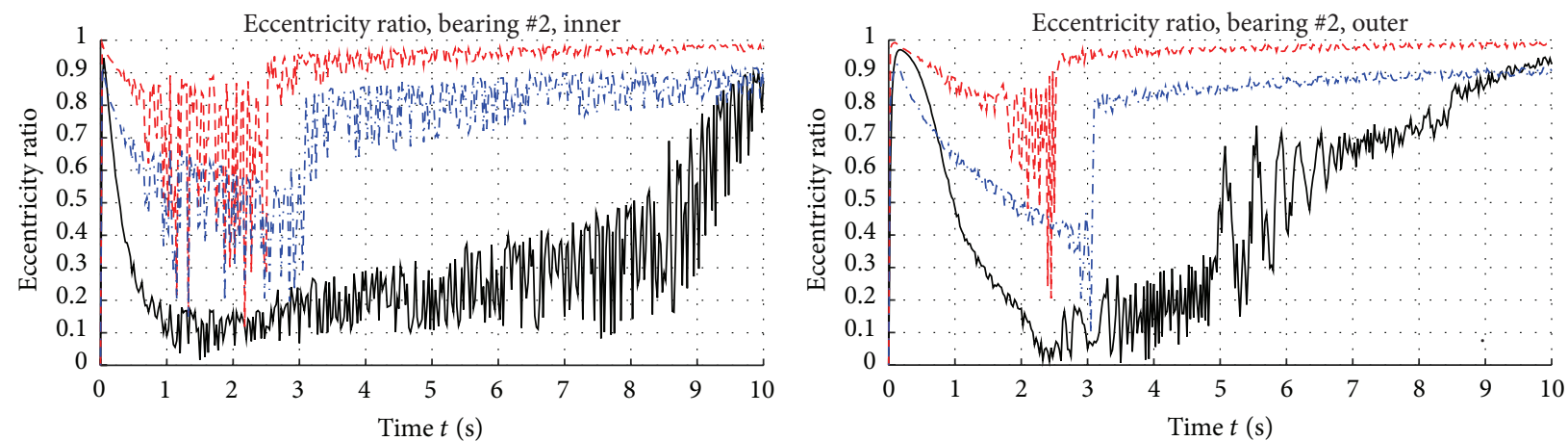

- Exact analytical
- - Approx. analytical
-. - Short bearing approx.

- Exact analytical

- - - Approx. analytical

-. - Short bearing approx.

(d)

FIGURE 13: System \#2: eccentricity ratio as a function of time for the different cases of simulation; (a) bearing \#1, inner film, (b) bearing \#1, outer film, (c) bearing \#2, inner film, and (d) bearing \#2, outer film.

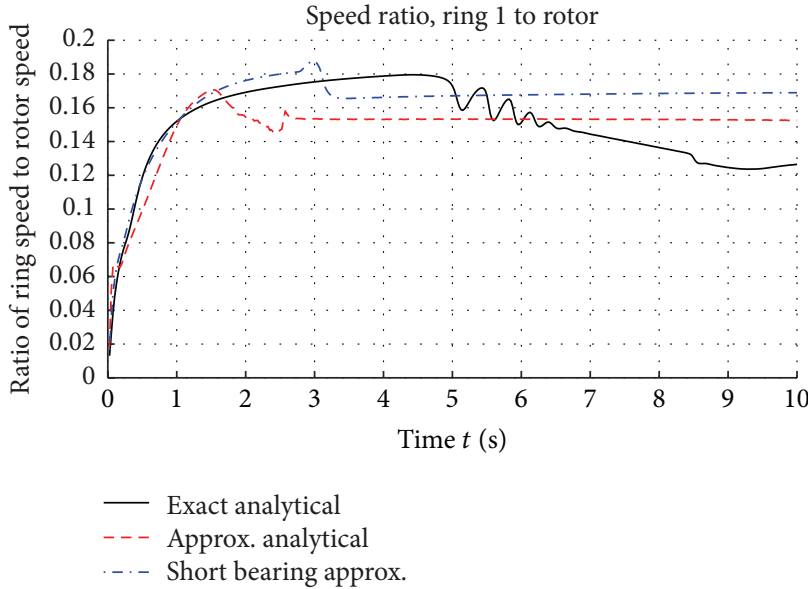

(a)

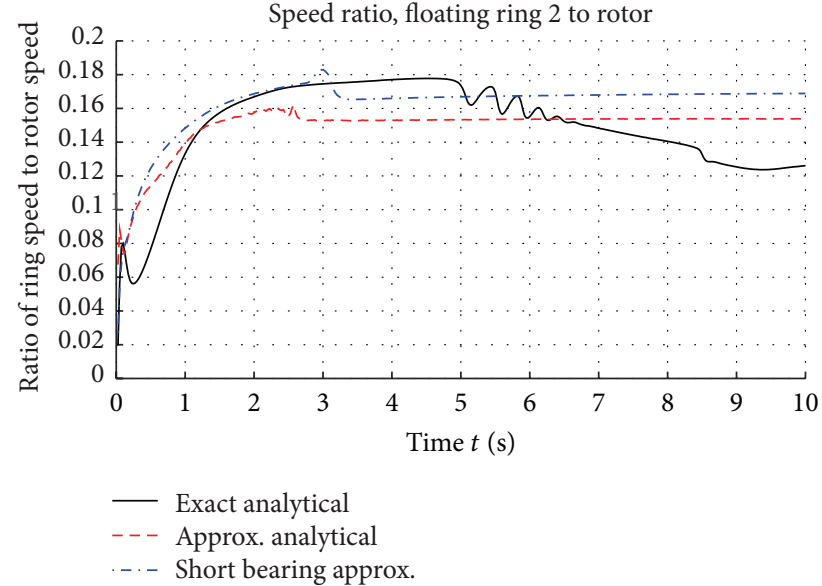

(b)

FIGURE 14: System \#2: ratio of the speed of the floating ring to the rotor's speed versus time for the different cases of simulation; (a) bearing $\# 1$ and (b) bearing \#2. 


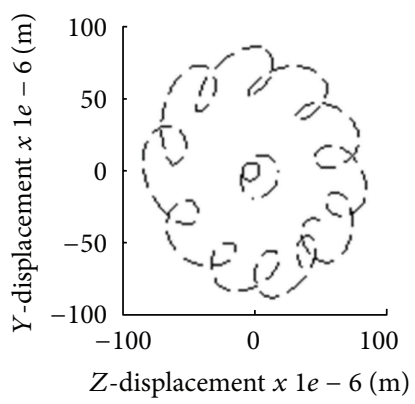

(a)

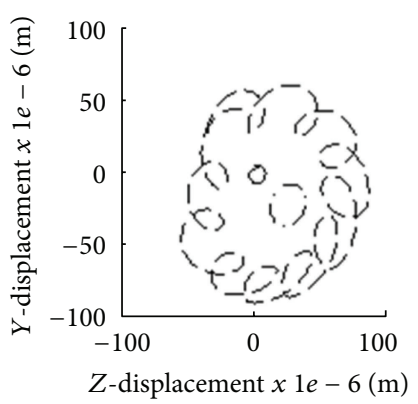

(e)

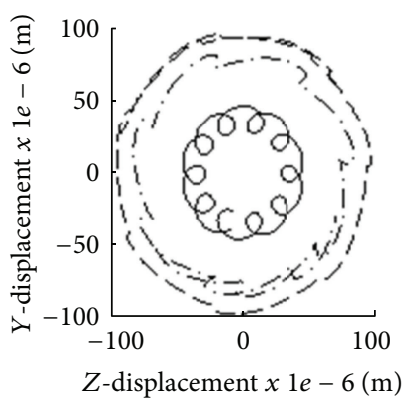

(b)

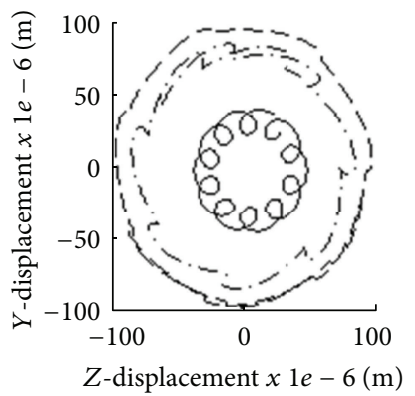

(f)

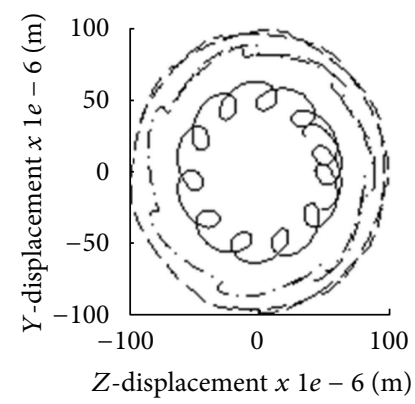

(c)

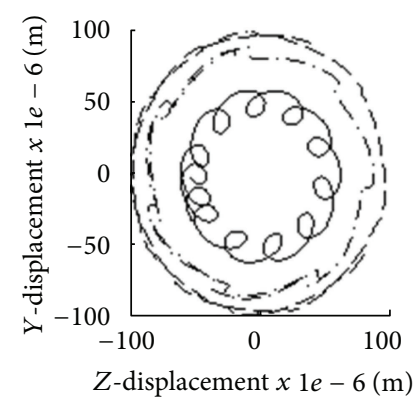

(g)

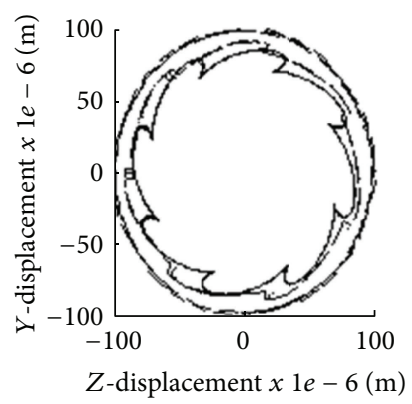

(d)

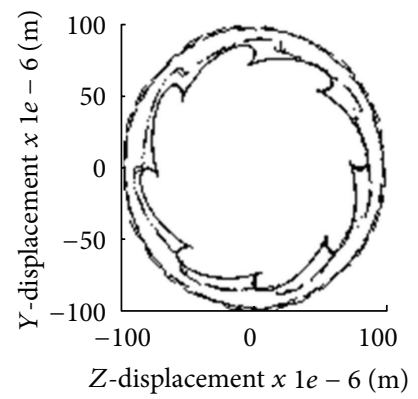

(h)

FIGURE 15: System \#2: orbits of the journal at bearing \#1 (a, b, c, d) and bearing \#2 (e, f, g, h) for the different cases of simulation at (a) and (e): $\Omega=40 \mathrm{kRPM}$; (b) and (f): $\Omega=80 \mathrm{kRPM}$; (c) and (g): $\Omega=120 \mathrm{kRPM}$; and (d) and (h) $\Omega=160 \mathrm{kRPM}$. Solid line: exact analytical solution (Case \#1), dashed line: approximate analytical solution (Case \#2), dash-dotted line: short bearing approximation (Case \#3), and dotted line: numerical solution (FDM) (Case \#4). See also Table 3 for the definition of the cases.

of $\Omega=40 \mathrm{kRPM}, \Omega=80 \mathrm{kRPM}, \Omega=120 \mathrm{kRPM}$, and $\Omega=160 \mathrm{kRPM}$ and for the duration of more than one driving period at each speed (see Figure 15) in order to cover the full repeated trajectory of the journals in time. On the contrary to the orbits of system \#1 (see Figure 9), the orbits of system \#2 declare nonlinear motion including additional harmonics; see Figure 15. Linear orbits are observed only at speed of $\Omega=40 \mathrm{kRPM}$ and only through the exact analytical solution and the short bearing approximation. All other speeds of $\Omega=180 \mathrm{kRPM}, \Omega=120 \mathrm{kRPM}$, and $\Omega=160 \mathrm{kRPM}$, exist on the range where instabilities occur and nonlinear orbits are observed through all three solutions. Further comments on the instability will be added after the time-frequency analysis.

The time-series presented in Figure 11 are decomposed in time and frequency domain through Short Time Fourier Transform (STFT). Figure 16 shows that the exact analytical solution (Figure 16(a)) and the numerical solution using FDM (Figure 16(d)) develop the same subharmonics in frequency and amplitude with the only difference noted in a subsynchronous component that is of slightly higher amplitude in the exact analytical solution. The sub-3 component is observed to be of equal amplitude and frequency characteristics in the exact analytical and the numerical solution using FDM. The approximate analytical solution using Bessel's functions and the short bearing approximation produce also the sub-3 component with very good agreement between them and a slight divergence regarding amplitude and the starting time of instability. Additionally, the short bearing approximation develops the sub-2 component for a very short time around $3 \mathrm{~s}$. In the current application, the amplitude of the components due to instability is high compared to that of the component of frequency synchronous to the driving frequency, noted as "syn." After these notes, the current bearing geometry is not assumed to form an efficient design and the various bearing simulations support this assessment. In system \#2, the length-to-diameter ratio of the fluid film is $L_{i, 1} /\left(2 R_{i, 1}\right)=0.56, L_{i, 2} /\left(2 R_{i, 2}\right)=0.56, L_{o, 1} /\left(2 R_{o, 1}\right)=0.74$, and $L_{o, 2} /\left(2 R_{o, 2}\right)=0.74$ for the corresponding fluid film, and the short bearing approximation cannot be compromised to the simulation demands. The exact analytical solution produces an instability of gradually increased amplitude and sets the response of the system near the radial clearance only towards the ending speed. Furthermore, it is in absolute agreement with the well-known numerical solution using FDM. On the contrary, the short bearing approximation and the approximate analytical solution produce a sub-3 component that sets the response of the system near radial clearance from the moment it is developed (even from low speeds at about $3 \mathrm{~s}$ ) until the ending speed; this would reject the design of the system regarding its bearing geometry (clearance, length, and diameter) and another set of bearing properties would be analysed further.

\section{Conclusions}

The nonlinear rotor dynamics of a high-speed system were studied in this work under proposed models for the floating ring bearings that incorporate exact and approximate 


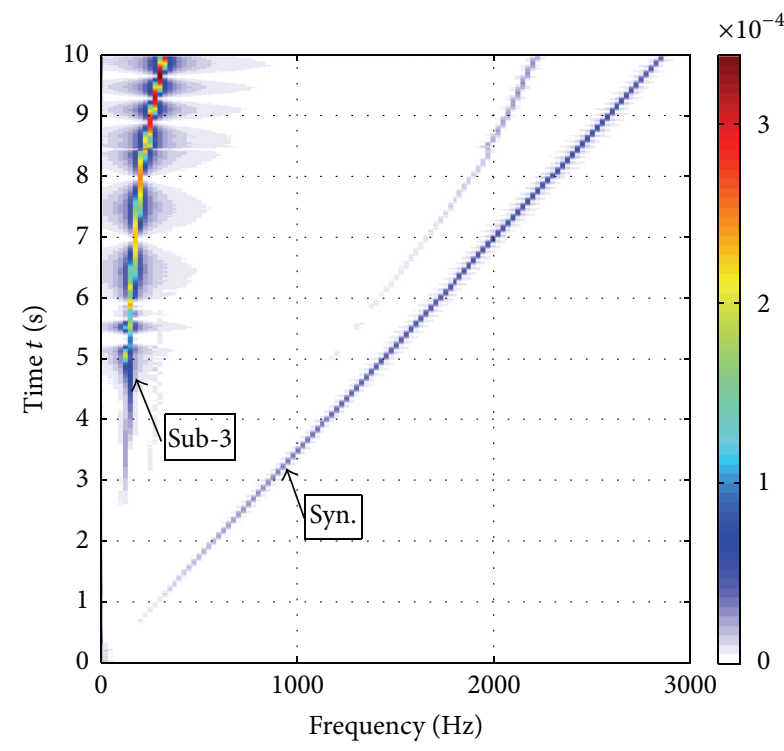

(a)

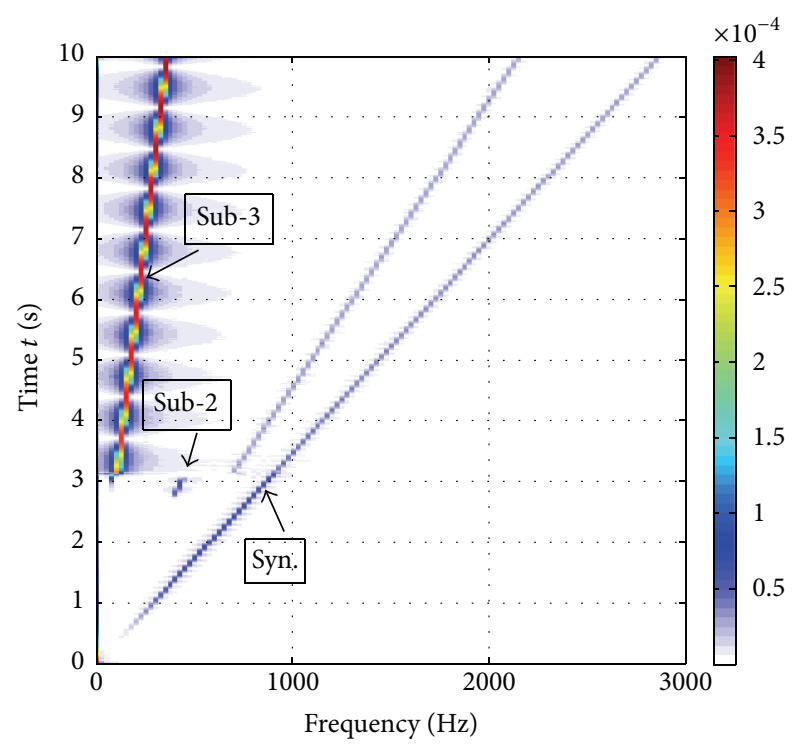

(c)

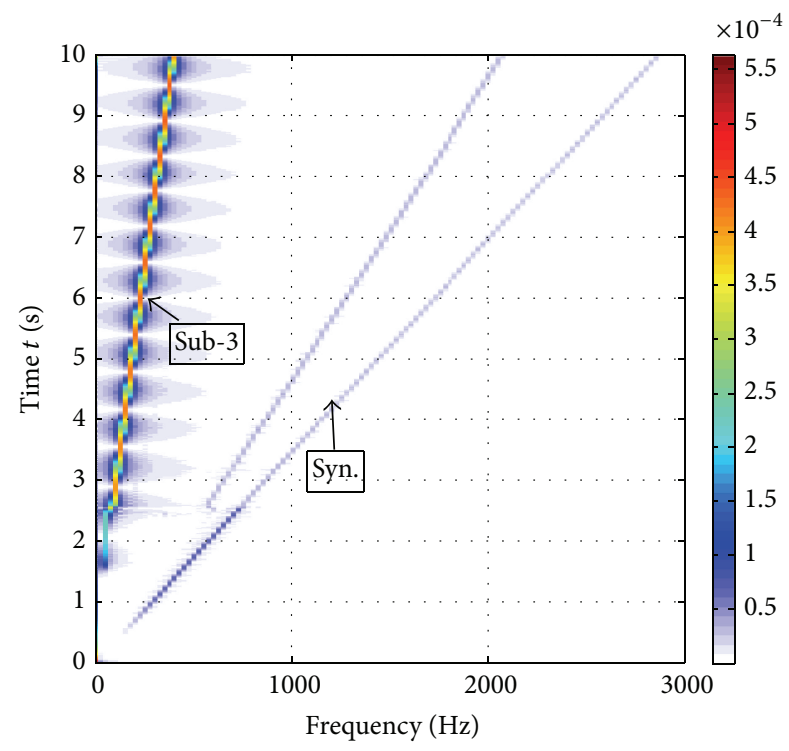

(b)

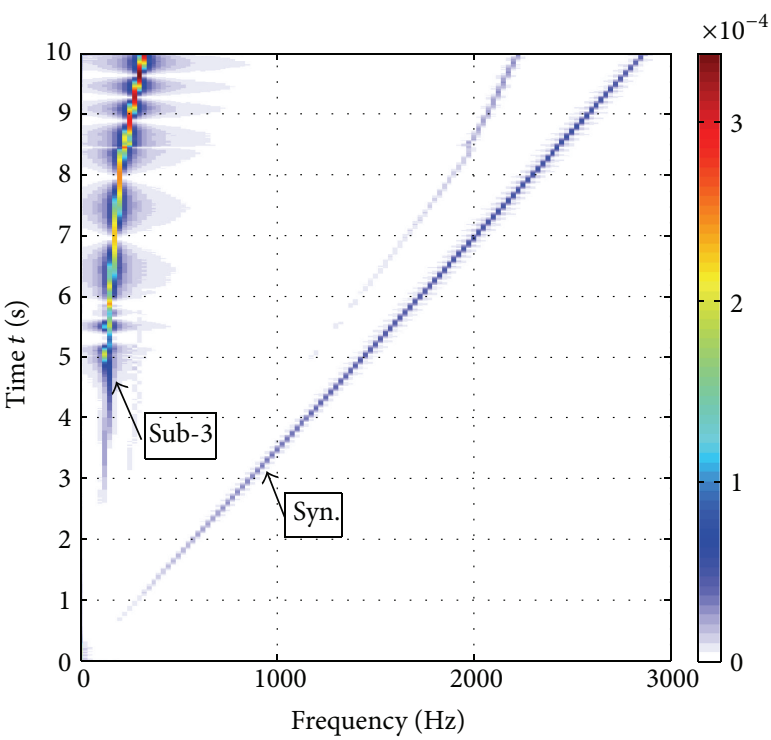

(d)

FIGURE 16: System \#2: time-frequency decomposition using STFT of the transient response of the journal of bearing \#1 for the simulation of (a) Case \#1, (b) Case \#2, (c) Case \#3, and (d) Case \#4.

analytical solutions of the Reynolds equation for a fluid film bearing of finite length. Additionally, two more bearing models, widely applied in the literature, the short bearing and the numerically solved finite-length bearing, have been presented in the paper to act as a reference for comparison of the proposed analytical solutions.

A rotor-bearing system was modeled consisting of a simple rigid rotor model and nonlinear floating ring bearings. Two systems of high-speed were studied regarding their dynamic response under the various bearing models.

The results showed that, in high-speed systems, the selection of the bearing model is of crucial importance and can have decisive role in the acceptance or rejection of a bearing geometrical configuration. The amplitude and frequency characteristics of the developed instabilities can significantly differ among the bearing theories used. The length-to-diameter ratio of the floating ring bearings used in turbosystems is not always such to justify short bearing approximation models and thus a new exact analytical bearing model is supposed to add further support to an efficient design.

The applicability of the exact analytical bearing model is furtherly supported by considering the evaluation time spent in the exact analytical bearing model and the numerical bearing model; although the numerical solutions are nowadays extremely fast and efficient, the exact analytical solution proposed is even faster compared to a traditional finite difference solution and not influenced much by the 
parameters of the solution regarding evaluation time and accuracy [26]. Furthermore, an exact analytical solution can form the basis for the entirely analytical treatment of the high-speed rotor dynamics that can hopefully result in analytical functions that confer the development of instabilities directly to the bearing geometrical properties. In this way, the numerous case studies for the exhaustive search of the most efficient bearing geometrical configuration can be substituted from a fast analytical treatment that will not only provide an efficient design but also contribute to the understanding of mechanisms of instabilities in such systems. The further development of the fluid film bearing exact analytical solution, avoiding sum of series, would benefit the applicability of the solution even more.

\section{Nomenclature}

Hellenic Letters

$$
\begin{aligned}
& \theta \text { : Coordinate at the circumferential } \\
& \text { direction of each oil film } \\
& \mu \text { : } \quad \text { Lubricant dynamic viscosity, used for } \\
& \text { bearing global analysis } \\
& \mu_{i}, \mu_{o} \text { : Dynamic viscosity of the lubricant at the } \\
& \text { inner and outer oil film, respectively } \\
& \tau_{i, 1}, \tau_{i, 2}: \text { Shear stresses of the inner oil film at the } 1 \text { st } \\
& \text { and } 2 \text { nd bearing, respectively } \\
& \tau_{o, 1}, \tau_{o, 2}: \text { Shear stresses of the outer oil film at the 1st } \\
& \text { and 2nd bearing, respectively } \\
& \varphi_{i, 1}, \varphi_{i, 2} \text { : Attitude angle of the inner oil film at the } \\
& \text { 1st and 2nd bearing, respectively } \\
& \varphi_{o, 1}, \varphi_{o, 2}: \text { Attitude angle of the outer oil film at the } \\
& \text { 1st and 2nd bearing, respectively } \\
& x \text { : Coordinate at the axial direction of each } \\
& \text { bearing } \\
& \psi_{Y} \text { : } \quad \text { Angle of tilting of the rotor in the vertical } \\
& \text { direction } \\
& \psi_{Z} \text { : } \quad \text { Angle of tilting of the rotor in the } \\
& \text { horizontal direction } \\
& \Omega \text { : } \quad \text { Rotating (spinning) speed of the shaft } \\
& \Omega_{r, 1}, \Omega_{r, 2} \text { : Rotating (spinning) speed of the floating } \\
& \text { ring at the } 1 \text { st and } 2 \text { nd bearing, } \\
& \text { respectively } \\
& \Omega_{\text {eff }} \text { : Effective rotational speed in a fluid film, } \\
& \text { used for global bearing analysis. }
\end{aligned}
$$

Latin Letters

$$
\begin{array}{ll}
c: & \begin{array}{l}
\text { Radial clearance, used for bearing global } \\
\text { analysis }
\end{array} \\
c_{i, 1}, c_{i, 2}: \begin{array}{l}
\text { Radial clearance at the inner oil film of the } \\
\text { 1st and } 2 \text { nd bearing, respectively }
\end{array} \\
c_{o, 1}, c_{o, 2}: \begin{array}{l}
\text { Radial clearance at the outer oil film of the } \\
\text { 1st and } 2 \text { nd bearing, respectively }
\end{array} \\
\mathrm{e}: \quad \begin{array}{l}
\text { Euler number } \\
\text { Eccentricity of a bearing; used for bearing } \\
\text { global analysis }
\end{array}
\end{array}
$$

$e_{1}, e_{2}: \quad$ Eccentricity of the journal in the inner oil film of the 1st and the 2nd bearing, respectively

$e_{r, 1}, e_{r, 2}: \quad$ Eccentricity of the ring in the outer oil film of the 1st and the 2 nd bearing, respectively

$f(\theta)$ : Homogenous solution of the Reynolds equation at the circumferential direction

$F_{g, 1}, F_{g, 2}: \quad$ Static forces applied to 1st and 2nd bearing respectively due to the gravity

$F_{Y, 1}, F_{Y, 2}$ : Resulting force of the inner oil film at the journal, at the vertical direction, and at the 1st and 2nd bearing, respectively

$F_{Z, 1}, F_{Z, 2}$ : Resulting force of the inner oil film at the journal, at the horizontal direction, and at the 1st and 2nd bearing, respectively

$F_{u, y, j}, F_{u, z, j}:$ Unbalance force applied from the $j$ th unbalance at the $j$ th unbalance level at the vertical and horizontal plane, respectively, $j=1,2,3,4$

$g: \quad$ Gravity acceleration

$g(x, \theta): \quad$ Homogenous solution of the Reynolds equation

$h: \quad$ Oil film thickness (trigonometric)

$J_{P}: \quad$ Equivalent polar moment of inertia of the system (rotor and wheels) reduced to the centre of mass of the system $\left(=J_{X X}\right)$

$I_{P, 1}, I_{P, 2}$ : Polar moment of inertia of the floating ring at the 1st and 2 nd bearing, respectively

$J_{T}$ : $\quad$ Equivalent moment of inertia of the system (rotor and wheels) reduced to the centre of mass of the system $\left(=J_{Z Z}=J_{Y Y}\right)$

$K$ : $\quad$ Number of eigenfunctions used for the analytical solution of the Reynolds equation

$L_{b}$ : Length of bearing, used for bearing global analysis

$L_{1}, L_{2}$ : $\quad$ Distance between 1st bearing and centre of mass of the system and between 2 nd bearing and centre of mass of the system

$L_{i, 1}, L_{i, 2}$ : Effective length of the inner fluid film at the 1st and 2nd bearing, respectively

$L_{o, 1}, L_{o, 2}$ : Effective length of the outer fluid film at the 1st and 2nd bearing, respectively

M: $\quad$ Equivalent mass of the system concentrated at the centre of mass of the system (rotor and wheels)

$m_{1}, m_{2}$ : Mass of the floating ring at the 1st and 2nd bearing, respectively

$N: \quad$ Number of Power Series terms

$P_{i, 1}, P_{i, 2}: \quad$ Developed pressure of the oil in the inner film at the 1st and 2nd bearing, respectively

$P_{o, 1}, P_{o, 2}: \quad$ Developed pressure of the oil in the outer film at the 1st and 2 nd bearing, respectively

$R: \quad$ Radius of a journal, used for bearing global analysis 
$R_{1}, R_{2}$ : Radius of rotor at the locations of the 1st and 2 nd bearing, respectively

$R_{i, 1}, R_{i, 2}$ : Inner radius of the floating ring at the 1st and 2 nd bearing, respectively

$R_{o, 1}, R_{o, 2}$ : Outer radius of the floating ring at the 1st and 2 nd bearing, respectively

$u_{j}$ : Magnitude of the $j$ th unbalance, $j=1,2,3,4$

$U_{j}$ : $\quad$ Distance at the axial direction of the system between the $j$ th unbalance plane and the centre of mass of the system (rotor and wheels), $j=1,2,3,4$

$u(x, \theta)$ : Particular solution of the Reynolds equation

$y_{\mathrm{CM}}$ : Vertical displacement of the centre of mass of the system (rotor and wheels)

$y_{1}, y_{2}$ : Vertical displacement of the rotor at the locations of the 1st and 2 nd bearing, respectively

$y_{r, 1}, y_{r, 2}$ : Vertical displacement of the ring at the 1st and 2 nd bearing, respectively

$z_{\mathrm{CM}}$ : Horizontal displacement of the centre of mass of the system (rotor and wheels)

$z_{1}, z_{2}$ : Horizontal displacement of the rotor at the locations of the 1st and 2nd bearing, respectively

$z_{r, 1}, z_{r, 2}$ : Horizontal displacement of the ring at the 1st and 2 nd bearing, respectively.

\section{Abbreviations}

ADI: Alternating Direction Implicit

BDF: Backward differentiation formula

CFD: Computational Fluid Dynamics

CM: Centre of mass of the rigid components of rotor and wheels

CW: Compressor wheel

DoE: Design of experiment

DoF: Degrees of freedom

FDM: Finite Difference Method

FEM: Finite Element Method

NDF: Numerical differentiation formula

ODE: Ordinary Differential Equation

PSM: Power Series Method

SLP: Sturm-Liouville problem

TW: Turbine wheel.

\section{Competing Interests}

The author declares that they have no competing interests.

\section{Acknowledgments}

The author would like to acknowledge Dr. Panagiotis Koutsovasilis (BorgWarner Turbosystems GmbH, 67292 Kirchheimbolanden, Germany) for the valuable discussions during the preparation of this work.

\section{References}

[1] B. Schweizer and M. Sievert, "Nonlinear oscillations of automotive turbocharger turbines," Journal of Sound and Vibration, vol. 321, no. 3-5, pp. 955-975, 2009.

[2] B. Schweizer, "Oil whirl, oil whip and whirl/whip synchronization occurring in rotor systems with full-floating ring bearings," Nonlinear Dynamics, vol. 57, no. 4, pp. 509-532, 2009.

[3] B. Schweizer, "Total instability of turbocharger rotors-physical explanation of the dynamic failure of rotors with full-floating ring bearings," Journal of Sound and Vibration, vol. 328, no. 1-2, pp. 156-190, 2009.

[4] B. Schweizer, "Dynamics and stability of turbocharger rotors," Archive of Applied Mechanics, vol. 80, no. 9, pp. 1017-1043, 2010.

[5] A. Boyaci, H. Hetzler, W. Seemann, C. Proppe, and J. Wauer, "Analytical bifurcation analysis of a rotor supported by floating ring bearings," Nonlinear Dynamics, vol. 57, no. 4, pp. 497-507, 2009.

[6] L. San Andres, J. C. Rivadeneira, K. Gjika, C. Groves, and G. LaRue, "Rotordynamics of small turbochargers supported on floating ring bearings-highlights in bearing analysis and experimental validation," ASME Journal of Tribology, vol. 129, no. 2, pp. 391-397, 2007.

[7] A. Tatara, "Vibration suppressing effect of floating bush bearings. Report of the Research Group of High-Speed Rotating Machinery," The Japan Society of Mechanical Engineers, vol. 72, pp. 1565-1569, 1969.

[8] M. Tanaka and Y. Hori, "Stability characteristics of floating bush bearings," ASME Journal of Lubrication Technology, vol. 94, no. 3, pp. 248-259, 1972.

[9] M. Tanaka, Y. Hori, and T. Nasuda, "A theoretical stability analysis of floating bush journal bearing," Proceedings of the Japan Society of Mechanical Engineers, vol. 750, no. 6, pp. 153-156, 1975.

[10] A. Tatara, "An experimental study of the stabilizing effect of floating-bush journal bearings," Bulletin of JSME, vol. 13, no. 61, pp. 858-863, 1970.

[11] K. Hatakenaka, M. Tanaka, and K. Suzuki, "A theoretical analysis of floating bush journal bearing with axial oil film rupture being considered," ASME Journal of Tribology, vol. 124, no. 3, pp. 494-505, 2002.

[12] W. Seemann, C. Proppe, and G. Knoll, "Multibody run-up simulation of turbocharger rotors with nonlinear modeled floating ring bearings," in Proceedings of the Research Association Engine/Turbomachinery, Semi-Annual Congress, Report No. R 542, Frankfurt am Main, Germany, 2008.

[13] A. Boyaci, D. Lu, and B. Schweizer, "Stability and bifurcation phenomena of Laval/Jeffcott rotors in semi-floating ring bearings," Nonlinear Dynamics, vol. 79, no. 2, pp. 1535-1561, 2014.

[14] P. Koutsovasilis, N. Driot, D. Lu, and B. Schweizer, "Quantification of sub-synchronous vibrations for turbocharger rotors with full-floating ring bearings," Archive of Applied Mechanics, vol. 85, no. 4, pp. 481-502, 2015.

[15] W. J. Chen and E. Gunter, Introduction to Dynamics of RotorBearing Systems, RODYN Vibration Analysis, Charlottesville, Va, USA, 2005.

[16] G. Adiletta, A. R. Guido, and C. Rossi, "Chaotic motions of a rigid rotor in short journal bearings," Nonlinear Dynamics, vol. 10, no. 3, pp. 251-269, 1996.

[17] G. Adiletta, A. R. Guido, and C. Rossi, "Nonlinear dynamics of a rigid unbalanced rotor in journal bearings. Part I: theoretical analysis," Nonlinear Dynamics, vol. 14, no. 1, pp. 57-87, 1997. 
[18] G. Adiletta, A. R. Guido, and C. Rossi, "Nonlinear dynamics of a rigid unbalanced rotor in journal bearings. Part II: experimental analysis," Nonlinear Dynamics, vol. 14, no. 2, pp. 157-189, 1997.

[19] R. Trippett, "Measured and predicted friction in floatingring bearings," SAE Transactions, vol. 95, pp. 1470-1476, 1986.

[20] R. J. Trippett and D. F. Li, "High-speed floating-ring bearing test and analysis," ASLE Transactions, vol. 27, no. 1, pp. 73-81, 1984.

[21] L. F. Shampine and M. W. Reichelt, "The MATLAB ODE suite," SIAM Journal on Scientific Computing, vol. 18, no. 1, pp. 1-22, 1997.

[22] L. Shampine, M. W. Reichelt, and J. Kierzenka, "Solving index-1 DAEs in MATLAB and simulink," SIAM Review, vol. 41, no. 3, pp. 538-552, 1999.

[23] D. Sfyris and A. Chasalevris, "An exact analytical solution of the Reynolds equation for the finite journal bearing lubrication," Tribology International, vol. 55, pp. 46-58, 2012.

[24] A. Chasalevris and D. Sfyris, "Evaluation of the finite journal bearing characteristics, using the exact analytical solution of the Reynolds equation," Tribology International, vol. 57, pp. 216234, 2013.

[25] A. Chasalevris, "Analytical evaluation of the static and dynamic characteristics of Three-Lobe journal bearings with finite length," ASME Journal of Tribology, vol. 137, no. 4, Article ID 041701, 16 pages, 2015.

[26] A. Chasalevris, "Finite length floating ring bearings: operational characteristics using analytical methods," Tribology International, vol. 94, pp. 571-590, 2016.

[27] A. Chasalevris, "Evaluation of the floating ring bearing characteristics using analytical methods," in Proceedings of the IFTOMM International Conference on Engineering Vibration (ICoEV '15), Ljubljana, Slovenia, September 2015.

[28] A. Chasalevris, "An investigation on the dynamics of highspeed systems using an analytical model for the floating ring bearings and the rotating shaft," in Proceedings of the IFTOMM International Conference on Engineering Vibration (ICoEV '15), Ljubljana, Slovenia, September 2015.

[29] T. Yamamoto and Y. Ishida, Linear and Nonlinear Rotordynamics, John Wiley and Sons, New York, NY, USA, 2001.

[30] The MathWorks, Matlab R2013b, The MathWorks, 2013.

[31] A. Polyanin and V. Zaitsev, Handbook of Exact Solutions for Ordinary Differential Equations, Chapman \& Hall/CRC, 2003. 


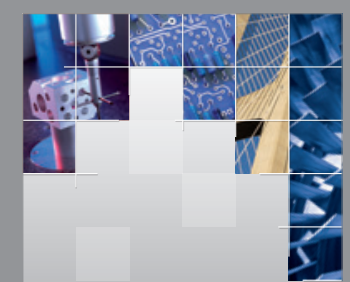

\section{Enfincering}
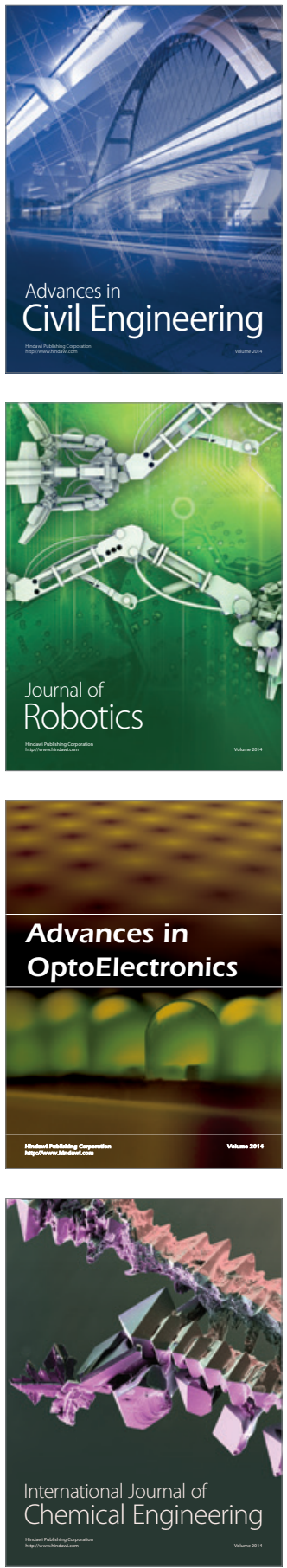

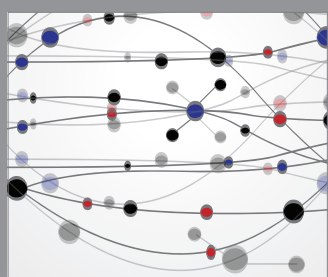

The Scientific World Journal

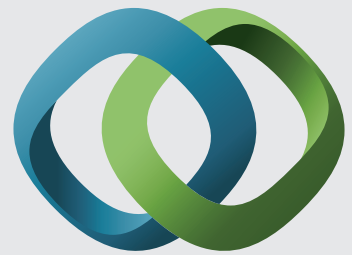

\section{Hindawi}

Submit your manuscripts at

http://www.hindawi.com
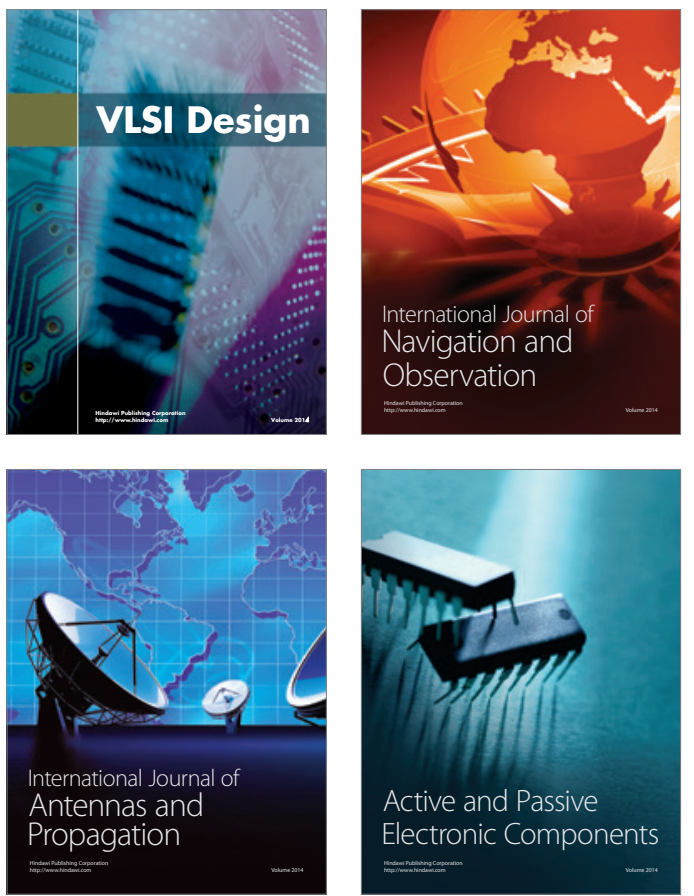
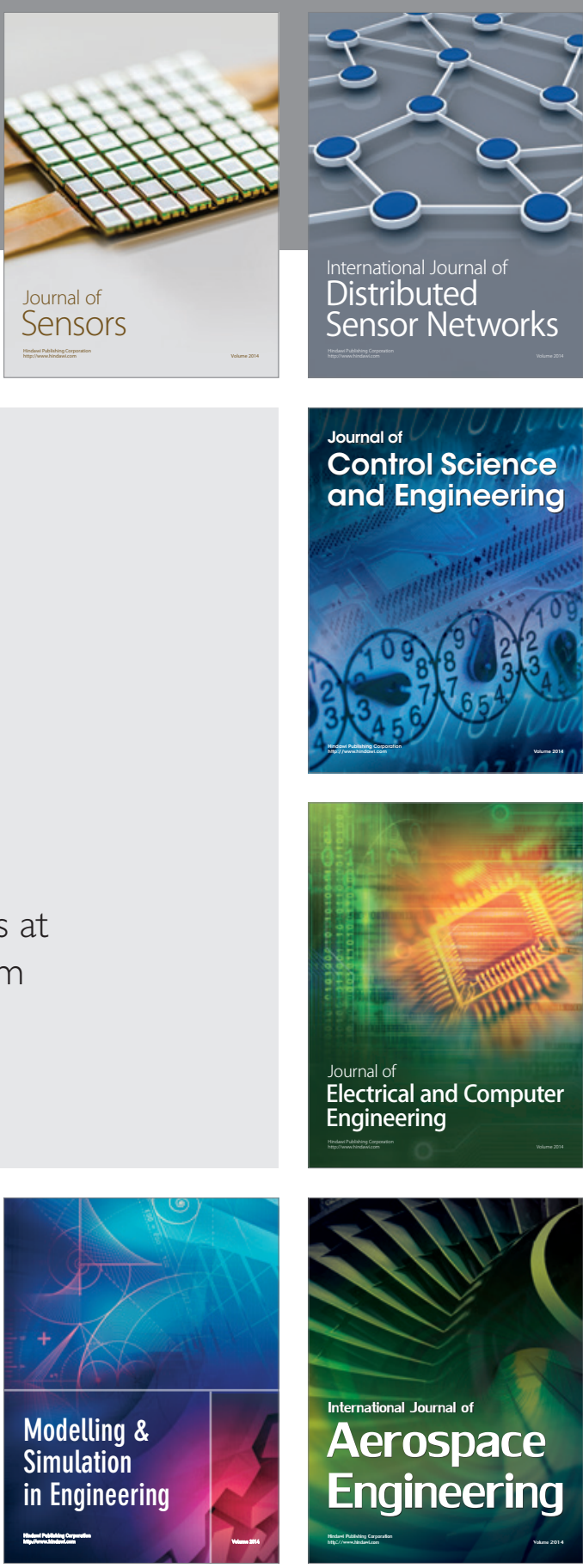

International Journal of

Distributed

Sensor Networks

Journal of

Control Science

and Engineering
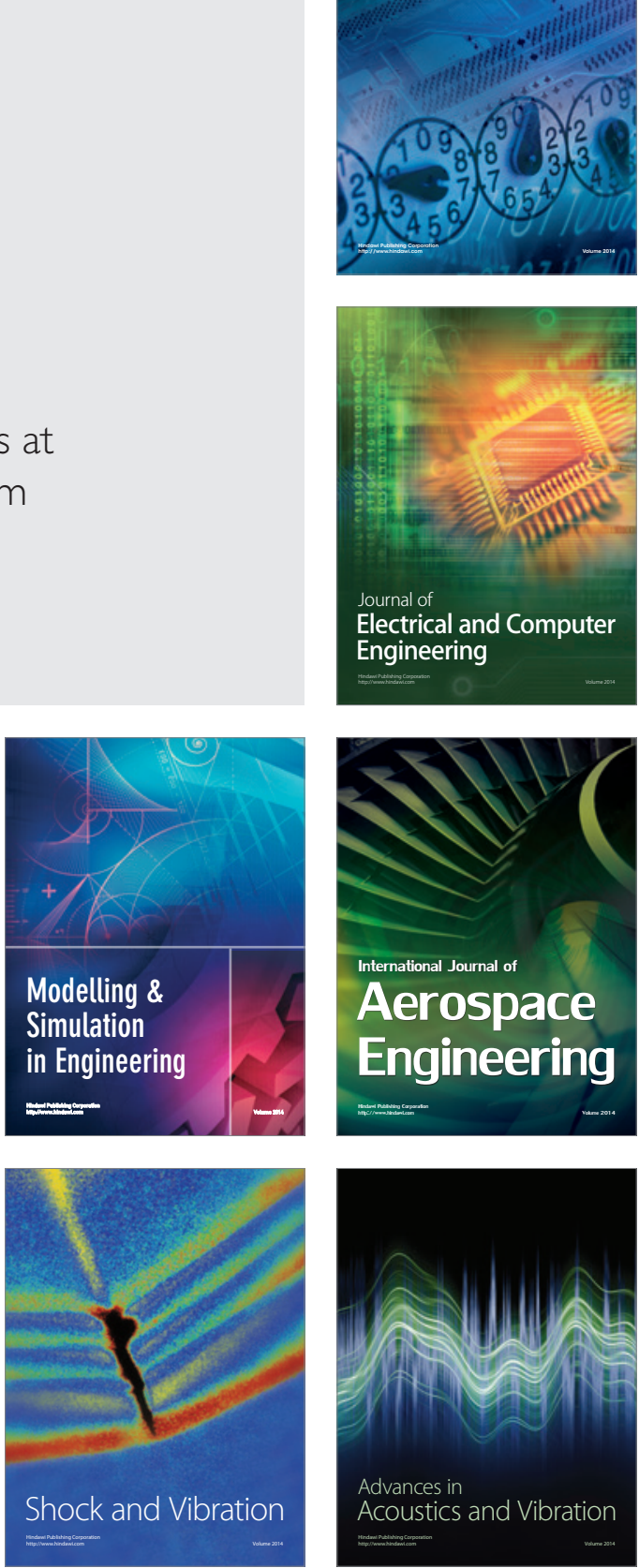